\title{
Fish Surveys of the Upper South Fork Judith River and its Tributaries Conducted during 2000
}

\author{
Bradley B. Shepard \\ Montana Fish, Wildlife and Parks \\ and \\ Montana Cooperative Fishery Research Unit \\ 1400 South $19^{\text {th }}$ \\ Bozeman, Montana 59718
}




\section{Table of Contents}

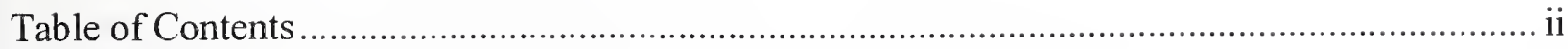

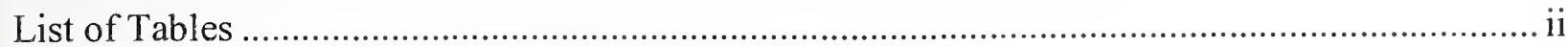

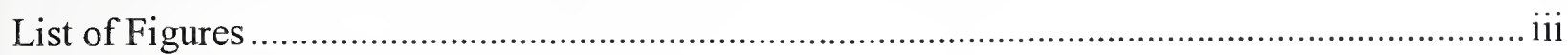

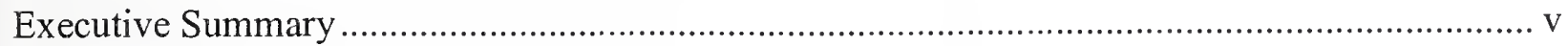

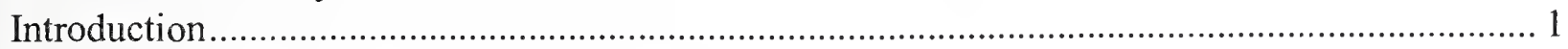

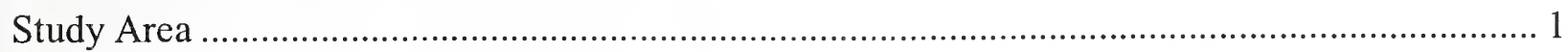

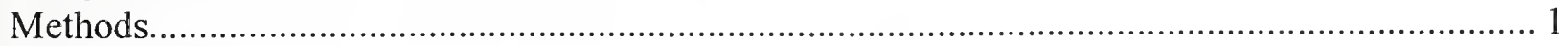

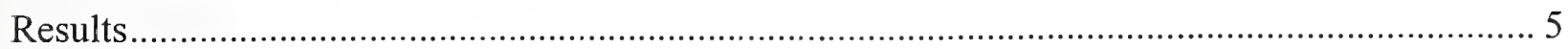

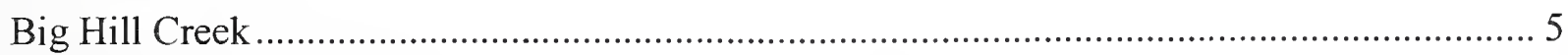

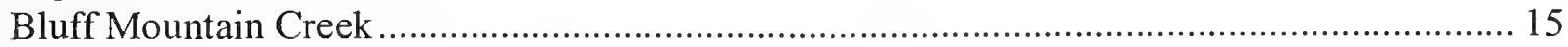

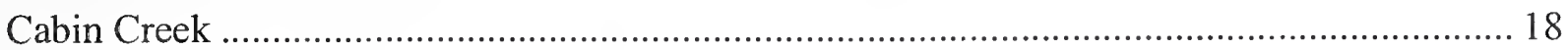

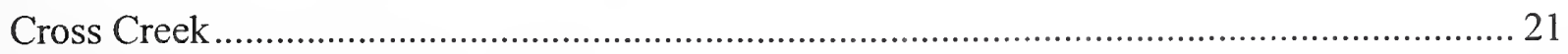

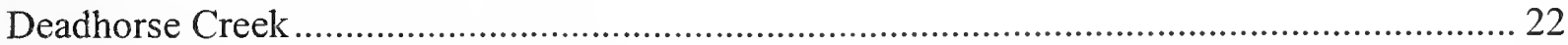

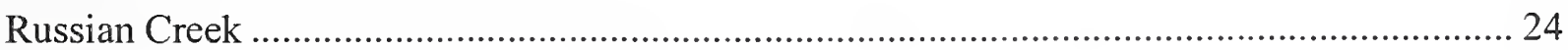

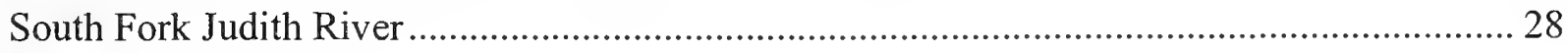

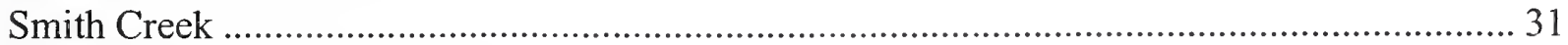

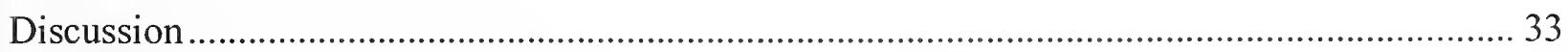

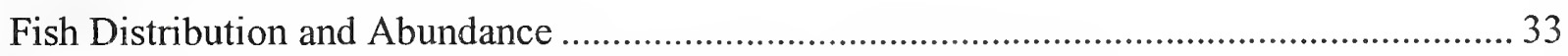

Recommendations for Conservation of Westslope Cutthroat Trout........................................... 34

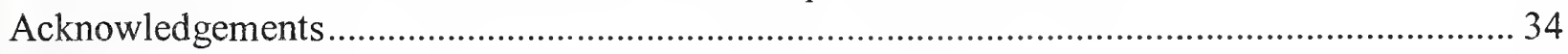

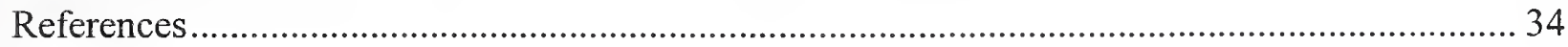

\section{List of Tables}

Table 1. Catch of westslope cutthroat trout (WCT) and rainbow trout (RB) per electrofishing pass, estimated number per section (standard error; S.E.), and section length (m) by stream, stream kilometer $(\mathrm{Km})$ and date during 2000 in the upper South Fork Judith River.

Table 2. Average length and weight (ranges) of fish captured during fish sampling in the upper South Fork Judith River and its tributaries by stream and stream kilometer.

Table 3. Streambed composition, frequency of small $(<150 \mathrm{~mm})$ and large $(>=150 \mathrm{~mm})$ inchannel and cross-channel woody debris per $\mathrm{km}$, and square meters of spawning habitat per $\mathrm{km}$ by stream, section, and date.

Table 4. Total length $(\mathrm{m})$, average length $(\mathrm{m})$, average width $(\mathrm{m})$, average depth $(\mathrm{cm})$, and average volume (cubic meters) of each habitat type and average thalweg depth $(\mathrm{cm})$ and residual pool volume (cubic meters) by stream and section.

Table 5. Rankings $(0=$ none or lowest; to $9=$ highest $)$ of instream cover, bank cover, bank stability, and pool quality by stream, section, and date. 


\section{List of Figures}

Figure 1. Map of upper South Fork Judith River drainage showing locations of thermographs, sites of fish abundance sampling (coded by fish species observed or captured), and stream kilometers (upstream from mouth) during 2000.

Figure 2. Map of streams sampled in upper South Fork Judith River drainage showing stream names and sample sites, by type of population abundance information collected........ 3

Figure 3. Average, minimum, and maximum water temperatures in Big Hill Creek immediately below the unnamed tributary during the summer of 2000 .

Figure 4. Length frequencies of westslope cutthroat trout captured in Big Hill Creek during 2000 .

Figure 5. Average, minimum, and maximum water temperatures in Bluff Mountain Creek just above its mouth during the summer of 2000 .....

Figure 6. Relative abundance (number of fish $75 \mathrm{~mm}$ and longer captured on the first electrofishing pass per $100 \mathrm{~m}$ of stream length) for westslope cutthroat (WCT), brook (EBT), and rainbow and rainbow/cutthroat trout hybrids $(\mathrm{RB}+\mathrm{HB})$ in four sections of Bluff Mountain Creek and three sections of an unnamed tributary by stream kilometer.

Figure 7. Length frequencies for westslope cutthroat trout in Bluff Mountain Creek (by stream kilometer up from mouth) and in three sections in a major unnamed tributary (TRIB) during July 2000.

Figure 8. Average, minimum, and maximum water temperatures in Cabin Creek just above its mouth during the summer of 2000 .

Figure 9. Relative abundance (number of fish $75 \mathrm{~mm}$ and longer captured on the first electrofishing pass per $100 \mathrm{~m}$ of stream length) for westslope cutthroat (WCT) and rainbow and cutthroat/rainbow trout hybrids $(\mathrm{RB}+\mathrm{HB})$ in eight sections of Cabin Creek by stream kilometer.

Figure 10. Length frequencies for westslope cutthroat trout in Cabin Creek (by stream kilometer up from mouth) during late June ( $\mathrm{km} 1.6$ and 3.2) and late July (km 0.8, 2.4, and 4.0 to

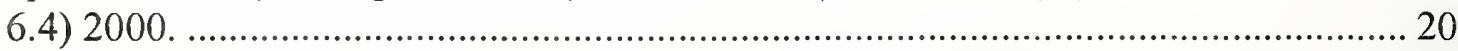

Figure 11. Length frequencies for westslope cutthroat trout in Cross Creek at stream kilometer 0.8 during late July 2000.

Figure 12. Average, minimum, and maximum water temperatures in Deadhorse Creek just above its mouth during the summer of 2000 ....

Figure 13. Relative abundance (number of fish $75 \mathrm{~mm}$ and longer captured on the first electrofishing pass per $100 \mathrm{~m}$ of stream length) for westslope cutthroat trout in six sections of Deadhorse Creek by stream kilometer.

Figure 14. Length frequencies for westslope cutthroat trout in six sections of Deadhorse Creek (by stream kilometer) during late July 2000.

Figure 15. Average, minimum, and maximum water temperatures in Russian Creek just below the South Fork Road crossing (top) and just above its mouth (bottom) during the summer of 2000 .

Figure 16. Relative abundance (number of fish $75 \mathrm{~mm}$ and longer captured on the first electrofishing pass per $100 \mathrm{~m}$ of stream length) for westslope cutthroat trout during 2000 in two sections of Russian Creek (left) and an unnamed tributary to Russian Creek (right) by stream kilometer. 
Figure 17. Length frequencies for westslope cut throat trout in two sections of Russian Creek (by stream kilometer) and its major unnamed tributary (Trib) during late June 2000. 27

Figure 18. Average, minimum, and maximum water temperatures in the South Fork Judith River below the mouths of Bluff Mountain Creek (top), Big Hill Creek (middle), and Corral Creek (bottom) during the summer of 2000 ...

Figure 19. Relative abundance (number of fish $75 \mathrm{~mm}$ and longer captured on the first electrofishing pass per $100 \mathrm{~m}$ of stream length) for westslope cutthroat trout (WCT) and rainbow trout $(\mathrm{RB}+\mathrm{HB})$ during 2000 in nine sections of the South Fork Judith River by stream kilometer.

Figure 20. Length frequencies for westslope cut throat trout in nine sections of the South Fork Judith River (by stream kilometer) during late June 2000.

Figure 21. Average, minimum, and maximum water temperatures in Smith Creek just above its mouth during the summer of 2000 .

Figure 22. Frequencies of relative abundance (number of fish $75 \mathrm{~mm}$ and longer per $100 \mathrm{~m}$ of stream length) for westslope cutthroat trout in allopatry in 489 sample sections that were a minimum of $50 \mathrm{~m}$ long within the streams of the upper Missouri River basin and a few streams in the upper Clark Fork basin of Montana. 


\section{Executive Summary}

This report summarizes fish survey information collected in the upper South Fork Judith River drainage in 2000. The purpose of the survey was to document the status of westslope cutthroat trout, Oncorhynchus clarki lewisi (WCT), within the upper South Fork drainage to help direct conservation efforts for WCT in this drainage. WCT were widely distributed in the upper South Fork Judith River basin and they were generally abundant where they occurred. Relative abundance for WCT generally ranged from 20 to 50 fish $75 \mathrm{~mm}$ and longer per $100 \mathrm{~m}$ of stream length and was as high as nearly 70 WCT per $100 \mathrm{~m}$ of stream. WCT in the South Fork Judith basin were found as far up into headwaters as stream size would support fish. Nonnative rainbow trout or fish identified as hybrids occupied the lower portions of Bluff Mountain and Cabin creeks, most of Smith Creek, and were also found in the South Fork Judith as far upstream as river km 19.3, just above Smith Creek. I am unsure how numerous rainbow trout are in the South Fork or how long they have been present in the upper portions of the basin, but their presence so far up the basin is a major concern for WCT conservation and restoration. The upper South Fork Judith River basin above Bluff Mountain Creek represents a good opportunity to restore a moderately large connected stream and river system for WCT. This area contains approximately $60 \mathrm{~km}$ of stream with approximately $40 \mathrm{~km}$ now inhabited by fish. Any attempt to conserve WCT in the upper South Fork will require construction of a fish barrier to eliminate the continuing invasion of brook and rainbow trout into the upper basin. Unfortunately, rainbow trout have already made their way up into the basin at least as far as Smith Creek. It also appears that WCT populations in several tributaries, including Russian and Cabin creeks, have already been slightly hybridized with either rainbow trout or Yellowstone cutthroat trout based on earlier genetic sampling. Fin clips taken for PINE genetic analyses during this study will provide more conclusive evidence for the genetic status of all WCT populations. Lastly, fish identified as either rainbow or hybrids between rainbow and WCT were captured in Bluff Mountain, Cabin, and Smith creeks, as well as the South Fork Judith. Thus, several decisions will have to be made regarding barrier location(s) and either management of existing slightly hybridized populations as conservation populations that could not be used as donors for other waters, or removal of existing hybridized populations and replacing these existing hybrid populations using genetically pure sources from other streams in the basin, either by natural dispersal or by active human intervention. 


\section{Introduction}

The Montana Cooperative Fishery Research Unit and Montana Fish, Wildlife and Parks (FWP) was asked by Region 4 of FWP and the Lewis and Clark National Forest to conduct fish surveys in waters of the upper South Fork Judith River, Montana to document the presence and status of westslope cutthroat trout (Oncorhynchus clarki lewisi; WCT). These surveys were done during 2000 in cooperation with Region 4 of FWP and the Lewis and Clark National Forest to develop a conservation plan for westslope cutthroat trout in the upper South Fork.

\section{Study Area}

The streams surveyed included Corral, Big Hill, Russian, Deadhorse, Cross, Cabin, Smith and Bluff Mountain creeks, as well as the main South Fork Judith River down to the mouth of Bluff Mountain Creek (Figure 1). Streams in the upper South Fork supported WCT, brook trout (Salvelinus fontinalis), and rainbow trout (Oncorhynchus mykiss), while the South Fork also supported mountain whitefish (Prosopium williamsoni) and mottled sculpin (Cottus bairdi).

\section{Methods}

A systematic sampling scheme was employed to estimate both the relative abundance and distribution of fishes and to quantify stream habitat characteristics. Sample sections ranging from 45 to $135 \mathrm{~m}$ were surveyed at a frequency of approximately every $0.8 \mathrm{~km}(0.5 \mathrm{mile})$ of stream length by single-pass electrofishing with backpack Smith-Root electrofishers (Models SR-12BP, SR-15B). At approximately $3.2 \mathrm{~km}$ (2 mile) intervals we conducted two-pass depletion population estimates (Van Deventer and Platts 1985; Figure 2). Population estimates were not made when few or no fish were captured during the first electrofishing pass.

Sample section lengths were usually at least 20 times, with most at least 30 times, the average wetted stream width. Lyons (1992) found that when stream lengths of 35 times the mean wetted width were sampled with a towed electrofishing unit, all species of fish in fish communities in warm water streams of Wisconsin were generally captured. Sample sites were referenced by mile above the stream's mouth, and later converted to kilometers above the mouth, and by latitude and longitude obtained from a global positions system (GPS; Garmin 12XL). Field GPS locations were input into an ARCVIEW (Version 3.2; 1999; Environmental Systems Research Institute, Inc.) event theme and projected on 1:100,000 stream hydrography layers. The field GPS locations were corrected to overlay the hydrography layer and stream kilometer locations when discrepancies existed between field GPS and mapped locations (Figures 1 and 2).

Length (total length in $\mathrm{mm}$ ) and weight (gm) were recorded for all captured salmonids. For twopass estimates to provide reasonable results, we assumed that field calculated probabilities of capture (calculated as $1-\left(C_{2} / C_{1}\right)$; where $C_{1}=$ number captured on the first pass, and $C_{2}=$ number captured on second pass) had to be 0.80 or higher (c.f., White et al. 1982; Riley and Fausch 1992). If field calculated probabilities of capture were less than 0.80 after two passes, additional electrofishing passes were usually made. Population estimates were calculated using a maximum likelihood estimator within the MICROFISH program (Van Deventer and Platts 1985) 
Digitized by the Internet Archive in 2017 with funding from Montana State Library

https://archive.org/details/fishsurveysofupp2001shep 


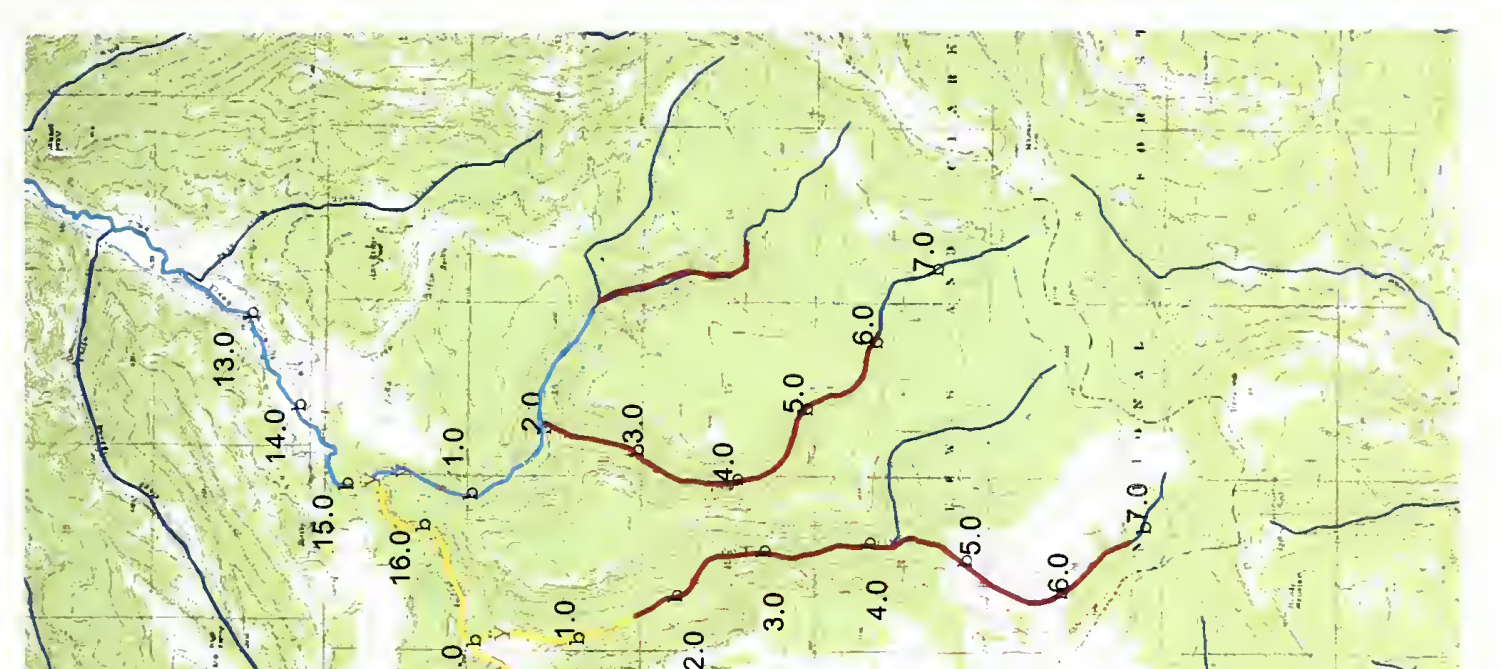

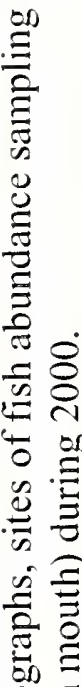
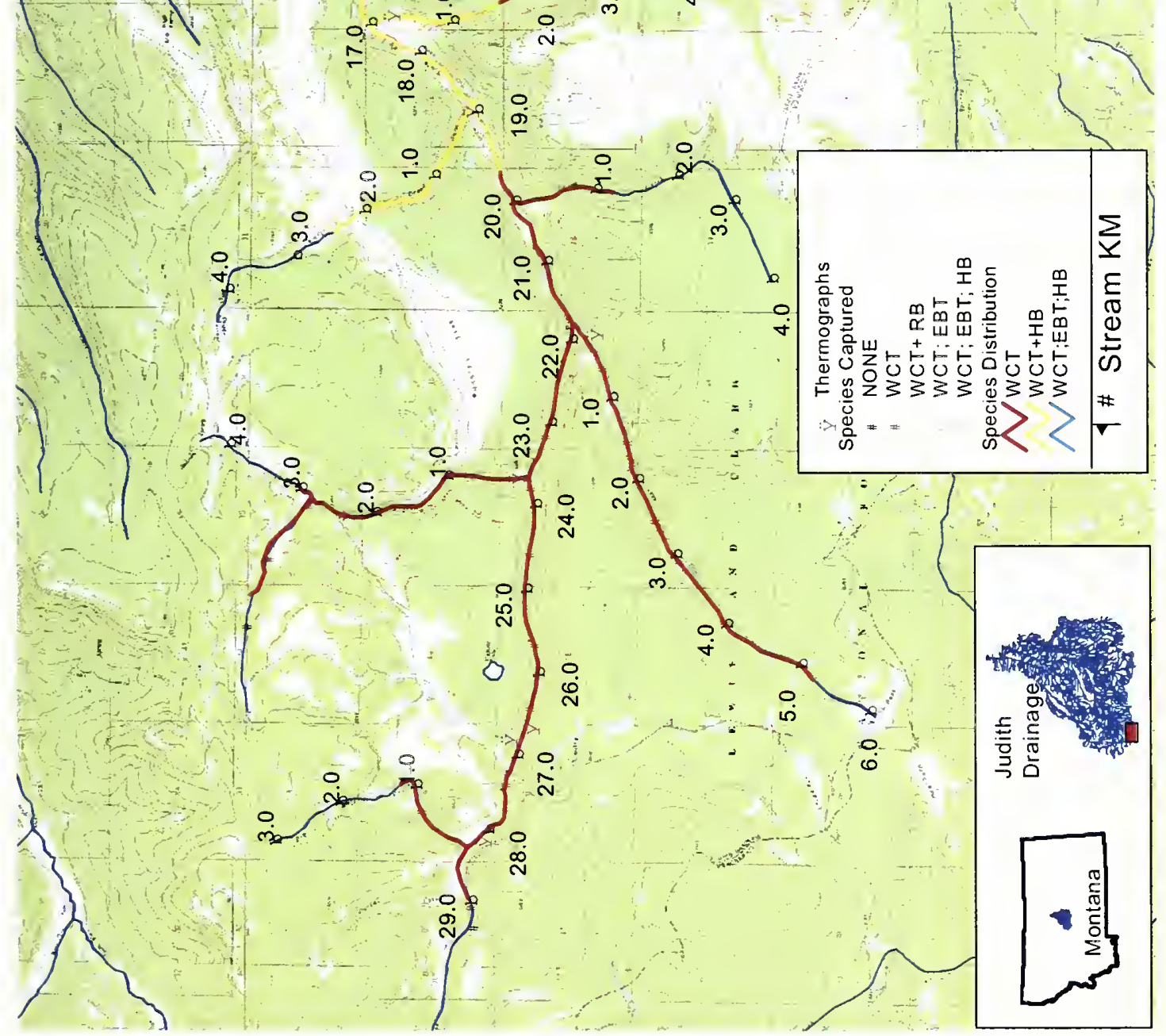

등

艺

.ิ气

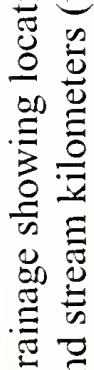

苛

: $\dot{\vec{\alpha}}$

吾

踣

$\Xi$

궁 룽

玟起

亏0

ธิ

है के

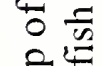

要㝴

它 



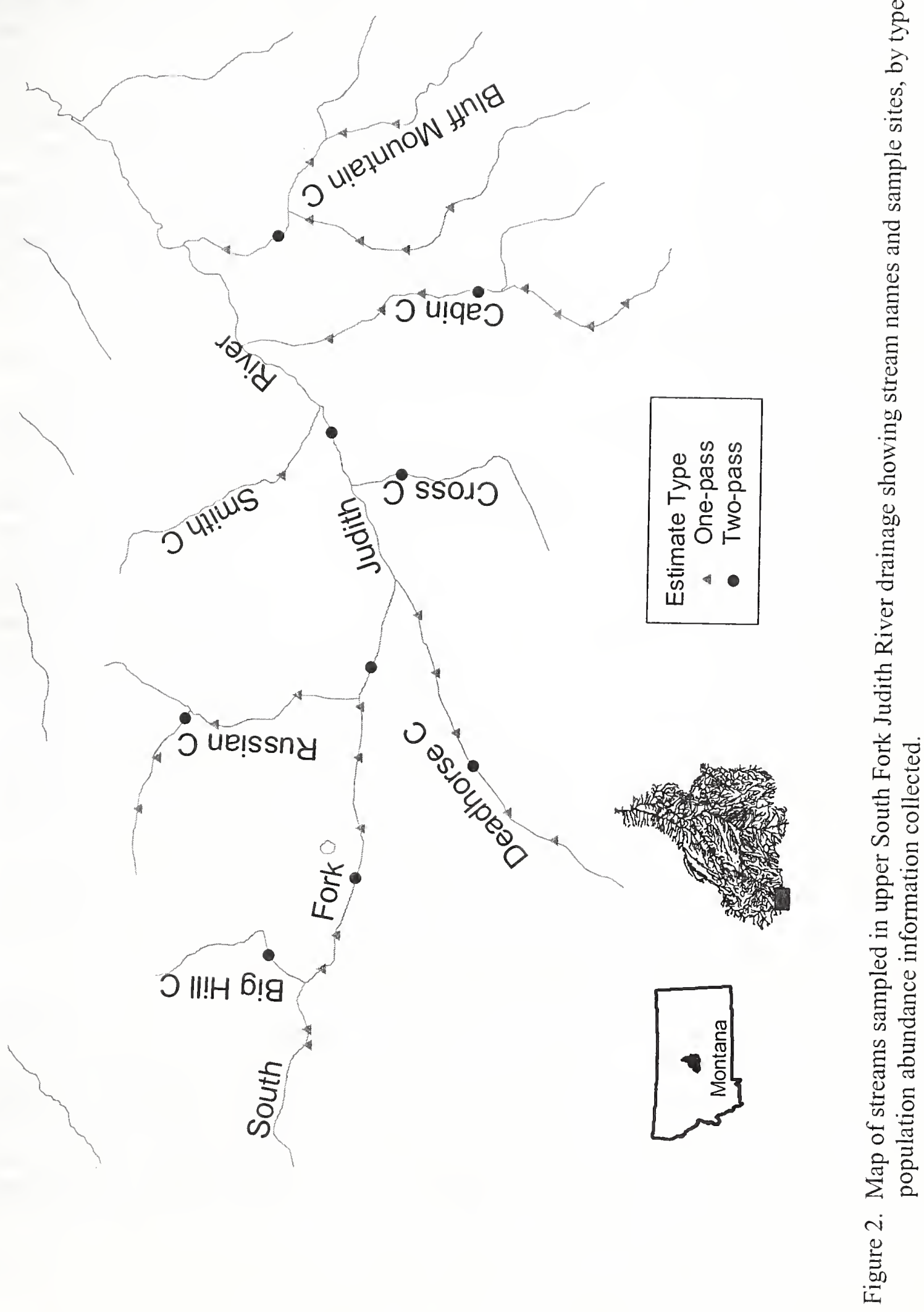


by species for fish $75 \mathrm{~mm}$ and longer. Population estimates of fish $75 \mathrm{~mm}$ and longer were also standardized per $100 \mathrm{~m}$ of stream length. Relative fish abundance was calculated as the number of fish $75 \mathrm{~mm}$ and longer per $100 \mathrm{~m}$ of stream length captured in the first electrofishing pass. Due to its large size, the South Fork Judith River below Deadhorse Creek could not be efficiently sampled using backpack electrofishers. Consequently, no sampling was conducted in the South Fork from Deadhorse Creek down river.

Fin samples from westslope cutthroat trout were taken for genetic analysis. Where possible, a sub-sample of westslope cutthroat trout captured at each sample site within a stream was represented in the genetic samples. The University of Montana Wild Salmon and Trout Genetics Laboratory, using Paired Interspersed Nuclear DNA Element-PCR (PINE) tests, will determine genetic status from these fin clips; however, results were not available at the time this report was completed. A listing of all tissue samples taken for genetic analyses and submitted to the Wild Salmon and Trout Genetics Laboratory are included in Appendix A.

Site level habitat surveys were conducted at $3.2 \mathrm{~km}$ ( 2 mile) intervals in sample sections where fish population estimates were made. The following information was collected for each macrohabitat type (pool, riffle or run) within a sample section: length of the macrohabitat type; wetted and channel width (width of normal bank-full channel), measured at a single location which represented an average width and depth of a habitat type; average depth, estimated by taking three depth measurements at equal distances across the single cross section where width was measured and dividing by 4; average maximum pool depth using 4 maximum (thalweg) depths were measured longitudinally down the channel and averaged; residual pool depth and volume were estimated using the average maximum depth of the pool minus the maximum depth of the adjacent downstream habitat unit, along with surface area of the pool for volume (Lisle 1987). Over the entire sample section the following information was collected: surface area of suitable spawning habitat (defined as patches of substrate dominated by material 10 to $30 \mathrm{~mm}$ which cover at least $\left.0.5 \mathrm{~m}^{2}\right)$; number of large $(>15 \mathrm{~cm}$ in diameter) and small $(\leq 15 \mathrm{~cm}$ in diameter) woody debris within the stream channel; number of large and small woody debris which span the stream channel; qualitative assessment of stream bank condition that ranked relative stability from low to high (and described the composition of the stream bank and the source of instability); qualitative assessment of instream cover which ranked the relative amount of instream cover from a low to high proportion of water volume with cover; qualitative assessment of bank overhead cover which ranked the amount of the water's surface which is covered or shaded; estimate of surficial streambed composition by size class in percentage by class; qualitative assessment of relative use of riparian areas by livestock or wildlife.

Continuously recording digital thermographs (models WTA08, Onset Computer Corporation, Pocasset, Massachusetts) were used to record water temperatures in Big Hill, Bluff Mountain, Cabin, Deadhorse, and Russian creeks and the South Fork (Figure 1). Model WTA08 thermographs were set to record temperatures every half hour. During mid-July thermographs were placed in well-mixed pools, shielded from direct solar radiation, from late June to mid- July and left to record stream temperatures until late September. Daily stream temperatures were summarized into daily average, maximum, and minimum recorded temperatures and graphed for each thermograph site by year. 


\section{Results}

\section{Big Hill Creek}

Big Hill Creek is a small tributary to the South Fork Judith River, entering the South Fork at about stream kilometer $(\mathrm{km}) 27.6$. Fish population and habitat surveys were conducted in Big Hill Creek just downstream from the South Fork Road, at the confluence with a small tributary to Big Hill Creek. This small tributary did not support fish and was dry at the end of the summer 2000 sampling period. Conifers dominate the riparian vegetation in this reach and stream habitat was generally excellent. No dispersal barriers were observed, although the culvert at the South Fork Road may cause passage problems during high flows. Average water temperatures in Big Hill Creek remained below $12 \mathrm{C}$, while maximum temperatures remained below $16 \mathrm{C}$, during the summer of 2000 (Figure 3).

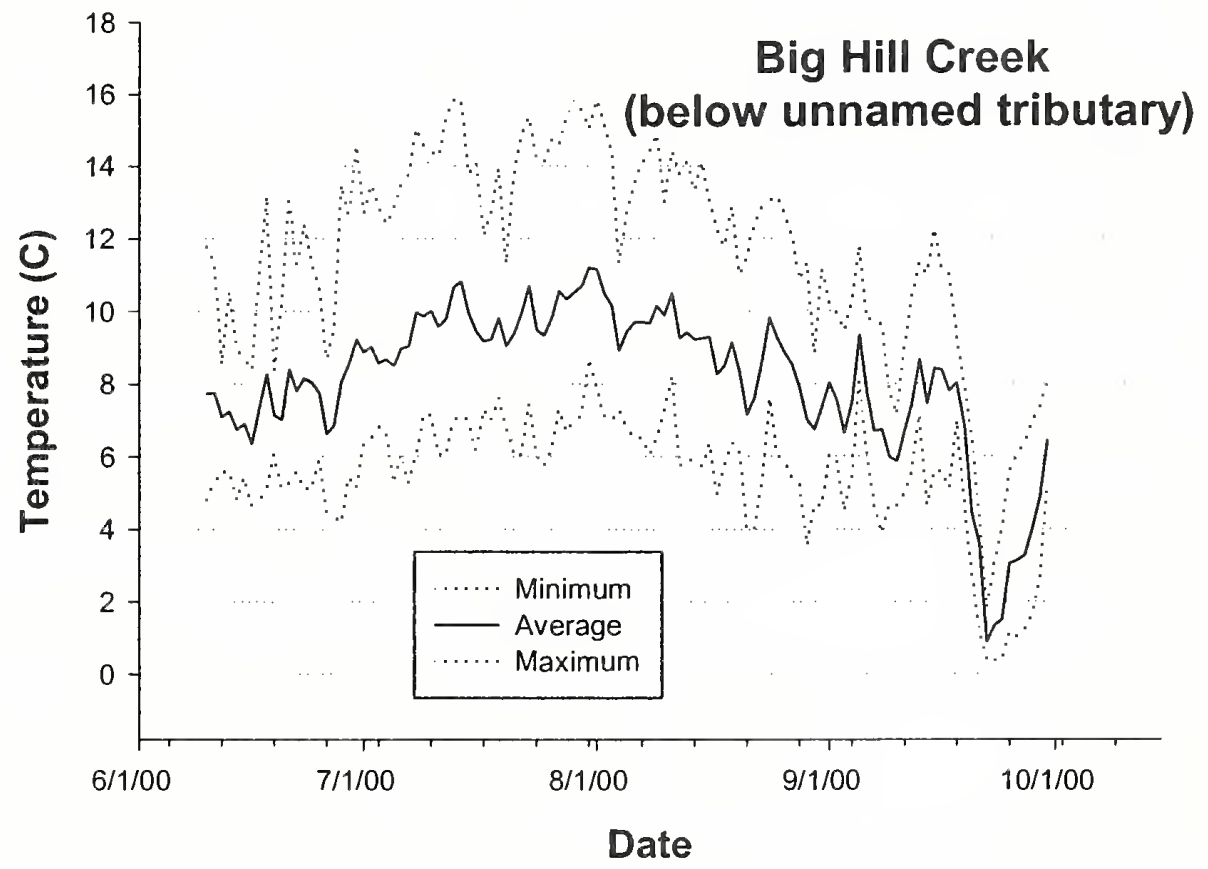

Figure 3. Average, minimum, and maximum water temperatures in Big Hill Creek immediately below the unnamed tributary during the summer of 2000.

Big Hill Creek supported only WCT. A genetics sample of 10 WCT taken by Mike Enk of the Lewis and Clark National Forest in August 1995 was 100\% pure WCT based on allozymes analysis (Tews et al. 2000). We collected fins from 25 WCT in June 2000 for genetic testing using the PINE test (Appendix A). No sculpins were observed. A $100 \mathrm{~m}$ sample section located at stream kilometer $(\mathrm{km}) 0.8$ supported an estimated 11 (SE not calculated due to all fish captured on pass 1), 32 (SE: 0.6), and 11 (SE not calculated due to all fish captured on pass 1) westslope cutthroat trout $<75 \mathrm{~mm}, 75-149 \mathrm{~mm}$, and $150 \mathrm{~mm}$ and longer, respectively (Table 1 and Appendix B). Captured westslope cutthroat trout averaged $109 \mathrm{~mm}$ (range: 46-217; Table 2; Figure 4). 
Table 1. Catch of westslope cutthroat trout (WCT) and rainbow trout (RB) per electrofishing pass, estimated number per section (standard error; S.E.), and section length $(\mathrm{m})$ by stream, stream kilometer $(\mathrm{Km})$ and date during 2000 in the upper South Fork Judith River.

\begin{tabular}{|c|c|c|c|c|c|c|c|c|}
\hline \multirow[b]{2}{*}{ Stream } & \multirow[b]{2}{*}{$\mathrm{Km}$} & \multirow[b]{2}{*}{ Date } & \multirow{2}{*}{$\begin{array}{l}\text { Section } \\
\text { length }(\mathrm{m})\end{array}$} & \multirow[b]{2}{*}{ Species } & \multicolumn{2}{|c|}{ Catch per pass } & \multirow[b]{2}{*}{ Estimate } & \multirow[b]{2}{*}{ S.E. } \\
\hline & & & & & 1 & 2 & & \\
\hline BIG HILL CR & 0.8 & $6 / 12 / 00$ & 100 & WCT & 40 & 3 & 43 & 0.5 \\
\hline BLUFF MOUNTAIN CR & 1.6 & $7 / 21 / 00$ & 100 & WCT & 29 & 5 & 34 & 1.0 \\
\hline CABIN CR & 3.2 & $6 / 25 / 00$ & 75 & WCT & 40 & 3 & 43 & 0.5 \\
\hline CROSS CR & 0.8 & $7 / 25 / 00$ & 90 & WCT & 31 & 3 & 34 & 0.6 \\
\hline DEADHORSE CR & 3.2 & $7 / 25 / 00$ & 75 & WCT & 20 & 7 & 29 & 3.0 \\
\hline RUSSIAN CR-W FK TRIB & 0.4 & $6 / 14 / 00$ & 100 & WCT & 23 & 5 & 28 & 1.1 \\
\hline \multirow[t]{2}{*}{ S FK JUDITH R } & 19.3 & $6 / 15 / 00$ & 135 & WCT & 71 & 6 & 77 & 0.7 \\
\hline & & & & $\mathrm{RB}$ & 2 & 0 & 2 & 0.0 \\
\hline S FK JUDITH R & 22.5 & $6 / 15 / 00$ & 100 & WCT & 57 & 8 & 64 & 1.0 \\
\hline S FK JUDITH R & 25.7 & $6 / 13 / 00$ & 105 & WCT & 50 & 4 & 54 & 0.6 \\
\hline
\end{tabular}

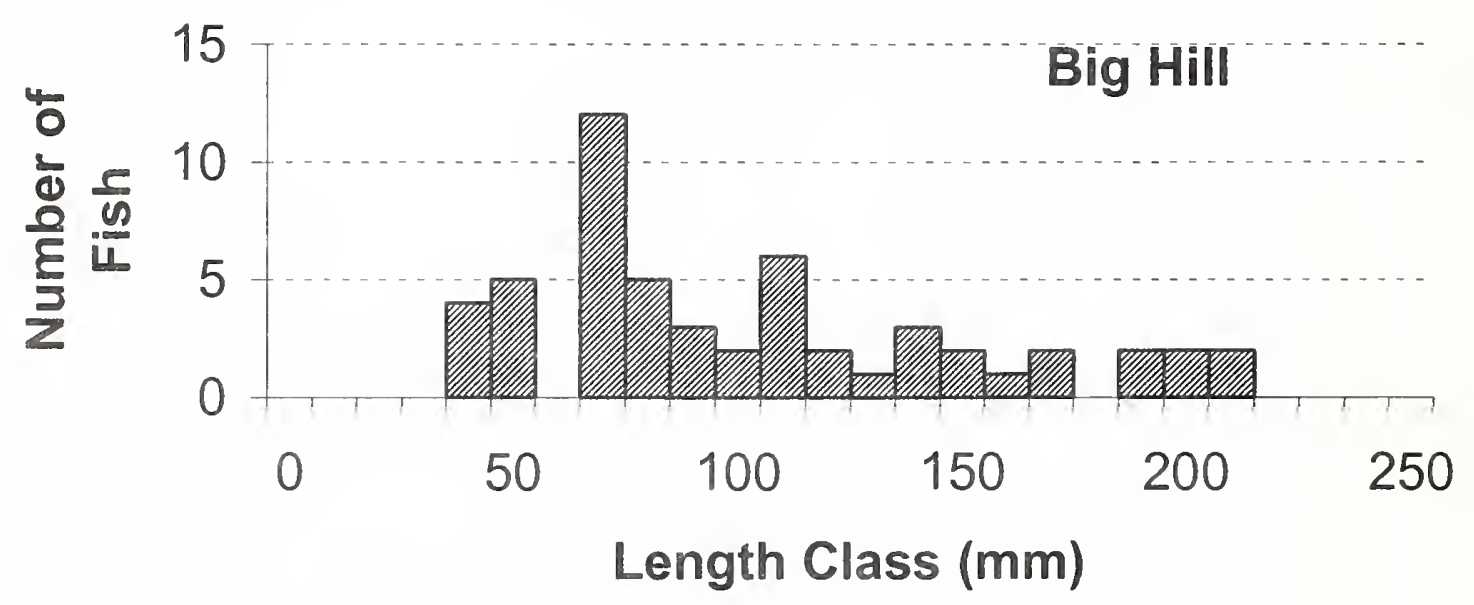

Figure 4. Length frequencies of westslope cutthroat trout captured in Big Hill Creek during 2000 .

A habitat survey conducted at $\mathrm{km} 0.8$ found that the streambed in Big Hill Creek at this location was comprised primarily of sand and gravels with sand and silt making up about $35 \%$ of the streambed's surface (Table 3). Small and large woody debris was relatively abundant in the stream channel with over $25 \%$ of the large debris crossing the entire wetted channel (Table 3 ). Spawning habitat appeared to be relatively abundant and was likely not limiting fish recruitment (Table 3 ). Pool habitats made up slightly over $40 \%$ by number and $30 \%$ by length of all habitat types (Table 4). Wetted width averaged $1.4 \mathrm{~m}$ and depth averaged $14.7 \mathrm{~cm}$ (Table 4). Pool volumes averaged about $0.7 \mathrm{~m}^{3}$. Pools had an average maximum depth of $18.5 \mathrm{~cm}$ and residual volume of $0.3 \mathrm{~m}^{3}$. Instream and bank cover, bank stability, and pool quality were all ranked 
Table 2. Average length and weight (ranges) of fish captured during fish sampling in the upper South Fork Judith River and its tributaries by stream and stream kilometer.

\begin{tabular}{lllll}
\hline STREAM & DThecies & $n$ & $\begin{array}{l}\text { Length } \\
\text { (range) }\end{array}$ & $\begin{array}{l}\text { Weight } \\
\text { (range) }\end{array}$ \\
\hline
\end{tabular}

\section{BIG HILL CR}

0.80

$$
06 / 12 / 2000 \quad \text { WCT }
$$

54

108.8

(46-217)

$(1-314)$

\section{BLUFF MOUNTAIN CR}

0.80

$$
\text { 07/24/2000 WCT }
$$

26

121.0

27.0

( 72- 212)

(3-99)

1.61

$\begin{array}{llccc}07 / 21 / 2000 & \text { RB } & 3 & 161.7 & 38.0 \\ 07 / 21 / 2000 & \text { EBT } & 3 & (147-182) & (34-42) \\ & & & (197.0 & - \\ 07 / 21 / 2000 & \text { WCT } & 38 & 145.1 & 30.7 \\ & & & (66-235) & (2-72)\end{array}$

2.41

$$
\text { 07/24/2000 WCT }
$$

15

$$
131.3
$$

26.0

(70-178)

(4- 62)

4.02

07/26/2000 WCT

10

136.6

(91- 174)

\section{BLUFF MOUNTAIN CR}

0.80

$\begin{array}{llcc}07 / 24 / 2000 & \text { EBT } & 1 & 185.0 \\ 07 / 24 / 2000 & \text { WCT } & 12 & (185-185) \\ & & 162.8 \\ & & (90-223)\end{array}$

1.61

$\begin{array}{llc}07 / 24 / 2000 \quad \text { WCT } & 9 & 141.2 \\ & & (85-203)\end{array}$

2.41

$\begin{array}{llc}07 / 24 / 2000 \quad \text { WCT } & 148.5 \\ & & (138-159)\end{array}$


Table 2. (continued).

\begin{tabular}{lll}
\hline STREAM & & \\
$\mathrm{Km}$ & Date & Species \\
\hline CABIN CR &
\end{tabular}

Length

(range)
Weight

(range)

0.80

\begin{tabular}{ccccc}
$07 / 26 / 2000$ & WCT & 18 & 128.3 & 30.8 \\
$07 / 26 / 2000$ & HB & \multirow{2}{*}{1} & $(54-223)$ & $(3-110)$ \\
& & & $(192.0$ & 75.0 \\
& & & $(192-192)$ & $(75-75)$
\end{tabular}

1.61

06/25/2000 WCT

10

133.2

29.5

(64-191)

( 5- 66)

2.41

07/25/2000 WCT

19

125.2

23.3

(51-187)

(5-61)

3.22

06/25/2000 WCT

43

138.1

27.3

(90-215)

(7- 120)

4.02

07/26/2000 WCT

24

143.8

33.4

(118-206)

(19-79)

4.83

07/26/2000 WCT

41

124.1

23.0

(66-190)

( 5- 67)

5.63

07/26/2000 WCT

35

135.5

29.8

(59-202)

(3-68)

6.44

07/26/2000 WCT

2

173.5

59.0

(147-200)

(38-80)

\section{CROSS CR}

0.80

07/25/2000 WCT

35

133.6

25.9

(59-188)

(4- 53)

\section{DEADHORSE CR}

0.80

$$
\text { 07/25/2000 WCT }
$$

8

159.9

48.5

(65-205)

(3-81)

1.61

07/25/2000 WCT

15

159.9

43.3

(113-226)

(12-82) 
Table 2. (continued).

\section{STREAM}

Length

Weight

$\mathrm{Km}$ Date

Species

(range)

(range)

2.41

$$
\text { 07/25/2000 WCT }
$$

23

130.8

(66-186)

30.2

(5-68)

3.22

07/25/2000 WCT

28

139.2

(61-208)

4.02

07/25/2000 WCT

18

124.3

(60-195)

4.83

07/25/2000 WCT

7

83.0

(56-136)

\section{RUSSIAN CR}

0.80

06/14/2000 WCT

6

129.3

(104- 180)

22.0

2.41

06/14/2000 WCT

22

126.9

27.2

(65-245)

(3-180)

RUSSIAN CR-W FK TRIB

0.40

06/14/2000 WCT

116.7

19.9

( 57- 199)

(3-66)

1.20

06/14/2000 WCT

9

147.4

33.1

(93-197)

( 7-63)

\section{S FK JUDITH R}

19.31

$\begin{array}{ll}06 / 15 / 2000 & \text { WCT } \\ 06 / 15 / 2000 & \text { RB }\end{array}$

77

165.4

84.5

( 81-292)

(2- 475)

2

230.0

230.0

(214-246)

(200-260)

22.53

06/15/2000 WCT

69

138.8

36.7

( 55- 229)

(2-106) 
Table 2. (continued).

\begin{tabular}{|c|c|c|c|c|c|}
\hline $\begin{array}{l}\text { STREAM } \\
\mathrm{Km}\end{array}$ & Date & Species & $\mathbf{n}$ & $\begin{array}{l}\text { Length } \\
\text { (range) }\end{array}$ & $\begin{array}{l}\text { Weight } \\
\text { (range) }\end{array}$ \\
\hline \multicolumn{6}{|l|}{23.33} \\
\hline & $06 / 13 / 2000$ & WCT & 34 & $\begin{array}{c}162.8 \\
(93-227)\end{array}$ & $\begin{array}{r}45.4 \\
(9-99)\end{array}$ \\
\hline \multicolumn{6}{|l|}{24.14} \\
\hline & $06 / 13 / 2000$ & WCT & 17 & $\begin{array}{c}152.1 \\
(98-211)\end{array}$ & $\begin{array}{c}49.1 \\
(11-118)\end{array}$ \\
\hline \multicolumn{6}{|l|}{24.94} \\
\hline & $06 / 13 / 2000$ & WCT & 34 & $\begin{array}{c}153.4 \\
(64-214)\end{array}$ & $\begin{array}{r}42.5 \\
(6-84)\end{array}$ \\
\hline \multicolumn{6}{|l|}{25.74} \\
\hline & $06 / 13 / 2000$ & WCT & 55 & $\begin{array}{c}157.1 \\
(62-227)\end{array}$ & $\begin{array}{c}61.1 \\
(4-170)\end{array}$ \\
\hline \multicolumn{6}{|l|}{26.55} \\
\hline & $06 / 13 / 2000$ & WCT & 45 & $\begin{array}{c}124.1 \\
(50-246)\end{array}$ & $\begin{array}{c}49.3 \\
(5-192)\end{array}$ \\
\hline \multicolumn{6}{|l|}{27.35} \\
\hline & $06 / 13 / 2000$ & WCT & 49 & $\begin{array}{c}133.7 \\
(53-217)\end{array}$ & $\begin{array}{r}31.6 \\
(2-97)\end{array}$ \\
\hline \multicolumn{6}{|l|}{28.32} \\
\hline & $06 / 12 / 2000$ & WCT & 6 & $\begin{array}{c}156.0 \\
(121-177)\end{array}$ & $\begin{array}{c}44.5 \\
(28-57)\end{array}$ \\
\hline \multicolumn{6}{|c|}{ SMITH CR } \\
\hline \multicolumn{6}{|l|}{0.80} \\
\hline & $07 / 25 / 2000$ & WCT & 13 & $\begin{array}{c}132.3 \\
(67-215)\end{array}$ & $\begin{array}{r}26.2 \\
(2-86)\end{array}$ \\
\hline & $07 / 25 / 2000$ & $\mathrm{HB}$ & 4 & $\begin{array}{c}153.0 \\
(118-205)\end{array}$ & $\begin{array}{c}43.5 \\
(14-97)\end{array}$ \\
\hline
\end{tabular}




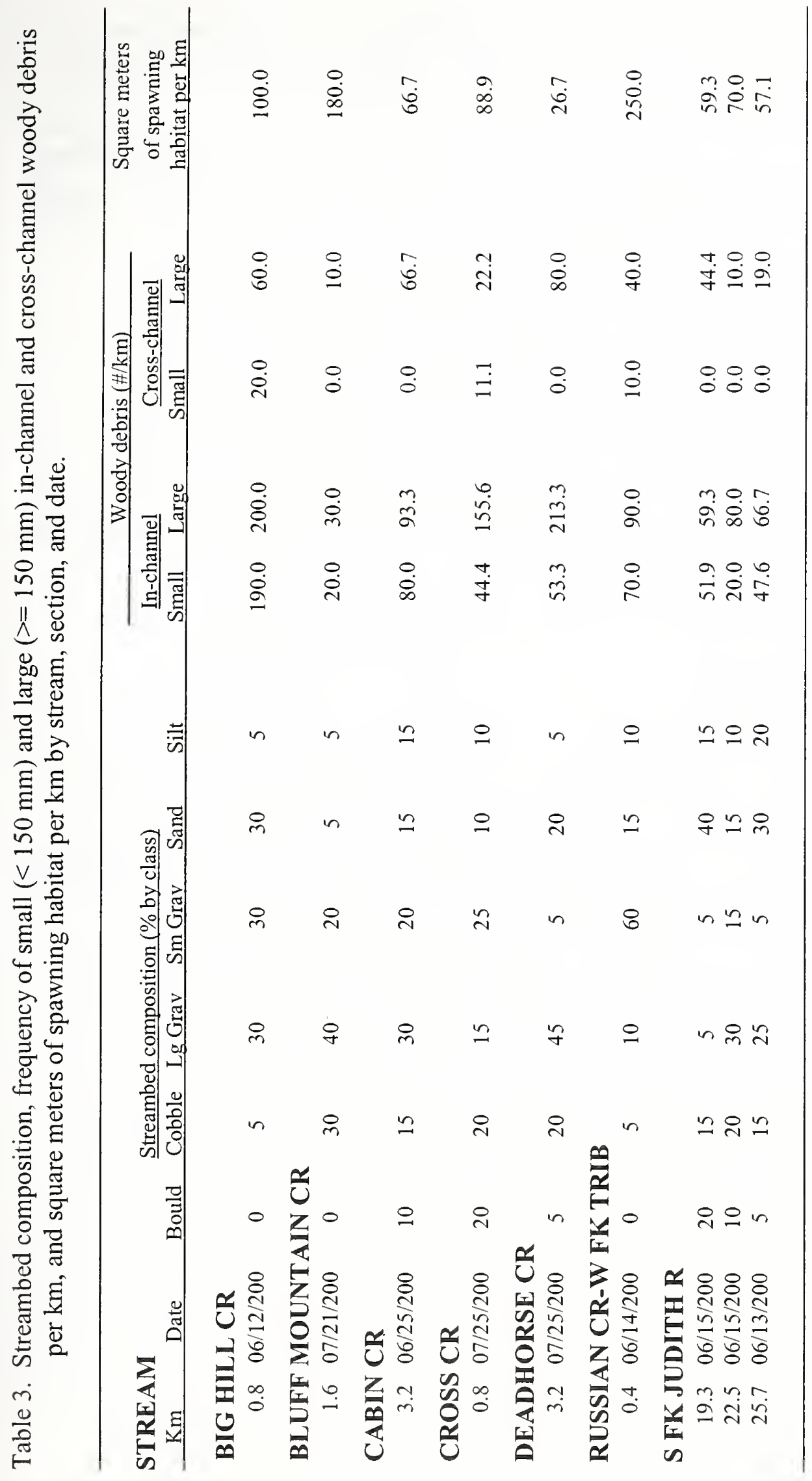


Table 4. Total length $(\mathrm{m})$, average length $(\mathrm{m})$, average width $(\mathrm{m})$, average depth $(\mathrm{cm})$, and average volume (cubic meters) of each habitat type and average thalweg depth $(\mathrm{cm})$ and residual pool volume (cubic meters) by stream and section.

\begin{tabular}{|c|c|c|c|c|c|c|c|c|c|}
\hline $\begin{array}{r}\text { STREAM } \\
\text { Date }\end{array}$ & $\begin{array}{c}\text { Habitat } \\
\text { type }\end{array}$ & $\mathrm{n}$ & $\begin{array}{l}\text { Total } \\
\text { length }\end{array}$ & $\begin{array}{l}\text { Average } \\
\text { length }\end{array}$ & $\begin{array}{l}\text { Average } \\
\text { width }\end{array}$ & $\begin{array}{l}\text { Average } \\
\text { Depth }\end{array}$ & Volume & $\begin{array}{c}\text { Average } \\
\text { thalweg } \\
\text { depth }\end{array}$ & $\begin{array}{c}\text { Residual } \\
\text { pool } \\
\text { volume }\end{array}$ \\
\hline
\end{tabular}

BIG HILL CR

$06 / 12 / 200$

STREAM KILOMETER:

0.8

$\begin{array}{rrrrrcrrr}\text { POOL } & 15 & 36.4 & 2.4 & 1.6 & 19.4 & 0.7 & 18.5 & 0.3 \\ \text { RIFFLE } & 14 & 49.1 & 3.5 & 1.3 & 8.7 & & & \\ \text { RUN } & 8 & 32.2 & 4.0 & 1.2 & 16.4 & & \end{array}$

$\begin{array}{lllll}\text { For Entire Section } & 117.7 & 3.2 & 1.4 & 14.7\end{array}$

\section{BLUFF MOUNTAIN CR}

\begin{tabular}{rcccccc}
$07 / 21 / 200$ & \multicolumn{3}{c}{ STREAM KILOMETER: } & & 1.6 \\
POOL & 4 & 11.3 & 2.8 & 2.3 & 22.0 & 1.6 \\
RIFFLE & 4 & 64.3 & 16.1 & 2.6 & 16.0 & \\
RUN & 2 & 16.8 & 8.4 & 2.4 & 19.0 &
\end{tabular}

$\begin{array}{lllll}\text { For Entire Section } \quad 92.3 & 9.2 & 2.4 & 19.0\end{array}$

\section{CABIN CR}

$06 / 25 / 200$

STREAM KILOMETER:

3.2

$\begin{array}{rcccccc}\text { POOL } & 6 & 15.3 & 2.6 & 2.3 & 23.0 & 1.4 \\ \text { RIFFLE } & 5 & 37.2 & 7.4 & 2.0 & 7.8 & \\ \text { RUN } & 1 & 6.0 & 6.0 & 2.2 & 21.0\end{array}$

For Entire Section

58.5

4.9

2.1

16.5 
Table 4. (continued).

\begin{tabular}{|c|c|c|c|c|c|c|c|c|c|c|}
\hline STREAN & $\begin{array}{l}\mathbf{1} \\
\text { Date }\end{array}$ & $\begin{array}{c}\text { Habitat } \\
\text { type }\end{array}$ & $\mathrm{n}$ & $\begin{array}{r}\text { Total } \\
\text { length }\end{array}$ & $\begin{array}{c}\text { Average } \\
\text { length }\end{array}$ & $\begin{array}{c}\text { Average } \\
\text { width }\end{array}$ & $\begin{array}{c}\text { Average } \\
\text { Depth }\end{array}$ & Volume & $\begin{array}{c}\text { Average } \\
\text { thalweg } \\
\text { depth }\end{array}$ & $\begin{array}{c}\text { Residual } \\
\text { pool } \\
\text { volume }\end{array}$ \\
\hline \multicolumn{11}{|c|}{ CROSS CR } \\
\hline \multicolumn{3}{|c|}{$07 / 25 / 200$} & \multicolumn{3}{|c|}{ STREAM KILOMETER: } & \multicolumn{3}{|c|}{0.8} & & \\
\hline & & POOL & 7 & 19.8 & 2.8 & 2.4 & 14.4 & 1.1 & & \\
\hline & & RIFFLE & 8 & 44.8 & 5.6 & 2.0 & 7.6 & & & \\
\hline & & RUN & 1 & 4.0 & 4.0 & 2.3 & 7.0 & & & \\
\hline & For Enti & ire Section & & 68.6 & 4.3 & 2.2 & 10.6 & & & \\
\hline
\end{tabular}

\section{DEADHORSE CR}

\begin{tabular}{rcccccr} 
07/25/200 & \multicolumn{9}{c}{ STREAM KILOMETER: } & 3.2 & \\
POOL & 8 & 25.4 & 3.2 & 2.0 & 29.3 & 2.1 \\
RIFFLE & 6 & 32.0 & 5.3 & 1.3 & 12.0 & \\
RUN & 2 & 14.0 & 7.0 & 2.1 & 14.0 &
\end{tabular}

$\begin{array}{lllll}\text { For Entire Section } & 71.4 & 4.5 & 1.7 & 20.9\end{array}$

\section{RUSSIAN CR-W FK TRIB}

\begin{tabular}{rcccccccr} 
06/14/200 & \multicolumn{7}{c}{ STREAM KILOMETER: } & \multicolumn{7}{c}{0.4} \\
POOL & 4 & 19.2 & 4.8 & 1.0 & 15.8 & 0.7 & 15.8 & 0.2 \\
RIFFLE & 5 & 71.3 & 14.3 & 1.1 & 7.6 & & \\
RUN & 1 & 2.2 & 2.2 & 1.3 & 21.0 & &
\end{tabular}

$\begin{array}{lllll}\text { For Entire Section } & 92.7 & 9.3 & 1.1 & 12.2\end{array}$


Table 4. (continued).

\begin{tabular}{|c|c|c|c|c|c|c|c|c|c|}
\hline $\begin{array}{r}\text { STREAM } \\
\text { Date }\end{array}$ & $\begin{array}{c}\text { Habitat } \\
\text { type }\end{array}$ & $\mathrm{n}$ & $\begin{array}{l}\text { Total } \\
\text { length }\end{array}$ & $\begin{array}{l}\text { Average } \\
\text { length }\end{array}$ & $\begin{array}{c}\text { Average } \\
\text { width }\end{array}$ & $\begin{array}{l}\text { Average } \\
\text { Depth }\end{array}$ & Volume & $\begin{array}{c}\text { Average } \\
\text { thalweg } \\
\text { depth }\end{array}$ & $\begin{array}{c}\text { Residual } \\
\text { pool } \\
\text { volume }\end{array}$ \\
\hline
\end{tabular}

\section{S FK JUDITH R}

\begin{tabular}{|c|c|c|c|c|c|c|c|c|}
\hline $06 / 15 / 200$ & STRI & $\mathbf{A M ~ K l}$ & METER & & 19.3 & & & \\
\hline POOL & 3 & 31.0 & 10.3 & 5.0 & 44.3 & 22.7 & & \\
\hline RIFFLE & 5 & 58.0 & 11.6 & 6.0 & 29.0 & & & \\
\hline RUN & 4 & 63.5 & 15.9 & 5.5 & 36.5 & & & \\
\hline For Entire Section & & 152.5 & 12.7 & 5.6 & 35.3 & & & \\
\hline $06 / 15 / 200$ & STRI & $\mathrm{AM} \mathrm{KI}$ & METER & & 22.5 & & & \\
\hline POOL & 2 & 17.0 & 8.5 & 3.3 & 40.0 & 11.6 & 45.0 & 5.9 \\
\hline RIFFLE & 4 & 61.0 & 15.3 & 4.4 & 21.3 & & & \\
\hline RUN & 2 & 14.0 & 7.0 & 4.0 & 19.5 & & & \\
\hline For Entire Section & & 92.0 & 11.5 & 4.0 & 25.5 & & & \\
\hline $06 / 13 / 200$ & STRI & AM KI & METER & & 25.7 & & & \\
\hline POOL & 5 & 31.0 & 6.2 & 3.0 & 33.2 & 6.9 & 37.8 & 2.5 \\
\hline RIFFLE & 6 & 43.4 & 7.2 & 2.2 & 16.8 & & & \\
\hline RUN & 2 & 25.0 & 12.5 & 2.5 & 24.5 & & & \\
\hline For Entire Section & & 99.4 & 7.6 & 2.6 & 24.3 & & & \\
\hline
\end{tabular}


Table 5. Rankings $(0=$ none or lowest; to $9=$ highest $)$ of instream cover, bank cover, bank stability, and pool quality by stream, section, and date.

\begin{tabular}{|c|c|c|c|c|c|}
\hline $\begin{array}{l}\text { STREAM } \\
\mathrm{Km} \text { Date }\end{array}$ & $\begin{array}{c}\text { Instream } \\
\text { cover }\end{array}$ & $\begin{array}{l}\text { Bank } \\
\text { cover }\end{array}$ & $\begin{array}{c}\text { Bank } \\
\text { stability }\end{array}$ & $\begin{array}{c}\text { Pool } \\
\text { quality }\end{array}$ & $\begin{array}{c}\begin{array}{c}\text { Riparian } \\
\text { use }\end{array} \\
\end{array}$ \\
\hline \multicolumn{6}{|l|}{ BIG HILL CR } \\
\hline $0.8 \quad 06 / 12 / 2000$ & 8 & 9 & 9 & 7 & 1 \\
\hline \multicolumn{6}{|c|}{ BLUFF MOUNTAIN CR } \\
\hline $1.607 / 21 / 2000$ & 4 & 7 & 6 & 3 & 1 \\
\hline \multicolumn{6}{|l|}{ CABIN CR } \\
\hline $3.206 / 25 / 2000$ & 7 & 8 & 8 & 7 & 3 \\
\hline \multicolumn{6}{|l|}{ CROSS CR } \\
\hline $0.8 \quad 07 / 25 / 2000$ & 5 & 6 & 7 & 7 & 3 \\
\hline \multicolumn{6}{|l|}{ DEADHORSE CR } \\
\hline $3.207 / 25 / 2000$ & 8 & 6 & 7 & 6 & 2 \\
\hline \multicolumn{6}{|c|}{ RUSSIAN CR-W FK TRIB } \\
\hline $0.4 \quad 06 / 14 / 2000$ & 6 & 7 & 5 & 4 & 3 \\
\hline \multicolumn{6}{|l|}{ S FK JUDITH R } \\
\hline $19.306 / 15 / 2000$ & 4 & - & - & - & - \\
\hline $22.5 \quad 06 / 15 / 2000$ & 7 & 7 & 7 & 5 & 2 \\
\hline $25.7 \quad 06 / 13 / 2000$ & 7 & 8 & 9 & 6 & 1 \\
\hline
\end{tabular}

high, while riparian use was rated as low (Table 5). Instream cover was primarily provided by woody debris.

\section{Bluff Mountain Creek}

Bluff Mountain Creek is a $6.5 \mathrm{~km}$-long tributary to the South Fork Judith. The upper $2 \mathrm{~km}$ of stream has a low to moderate gradient and flows through dense lodge pole pine forest. Fish habitat is marginal and no fish were captured above stream $\mathrm{km} \mathrm{4.8.} \mathrm{Below} \mathrm{km} \mathrm{4.8,} \mathrm{the} \mathrm{valley}$ bottom is much more confined, the channel gradient increases, and the quality of pool habitats improves. Many high quality pools are formed at the base of bedrock outcroppings. The headwaters of Bluff Mountain Creek had previously been logged, but the riparian zone was not harvested. No barriers to fish dispersal were observed in Bluff Mountain Creek; however, Tews et al. (2000) identified that a series of cascades may be a barrier to upstream fish movement. Due to its confined valley bottom and numerous bedrock outcroppings, there are many potential barrier construction sites. Bluff Mountain Creek has one unnamed tributary that enters between stream $\mathrm{km} 2.5$ and 3.0. Fish distribution and relative abundance was assessed at stream kilometers $0.8,1.6,2.4$, and 4.0 and fish population estimate and habitat surveys were conducted at $\mathrm{km}$ 1.6. Average water temperatures in lower Bluff Mountain Creek remained below $13 \mathrm{C}$, while maximum water temperatures remained below $15 \mathrm{C}$ (Figure 5). 


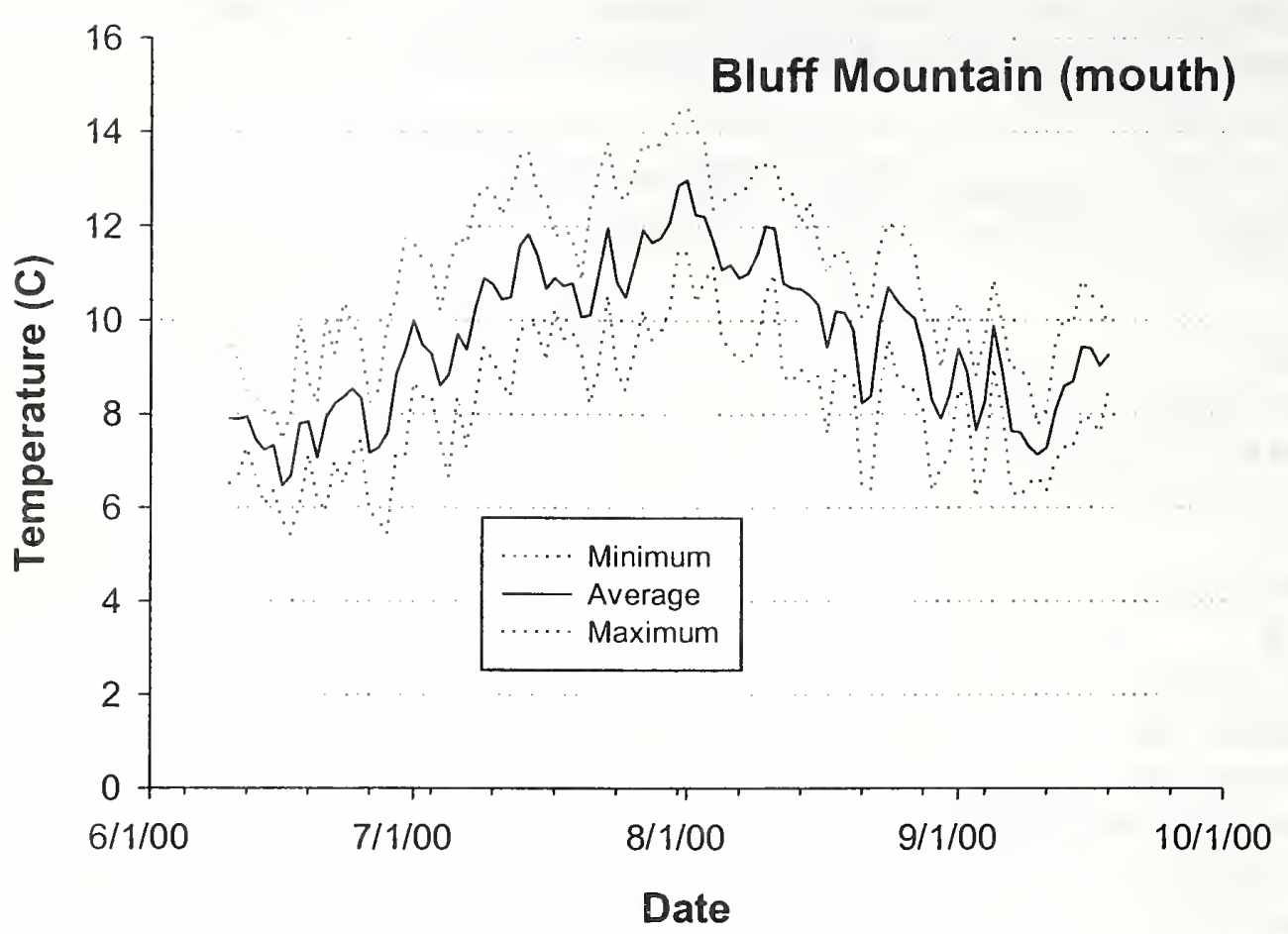

Figure 5. Average, minimum, and maximum water temperatures in Bluff Mountain Creek just above its mouth during the summer of 2000.

The major unnamed tributary to Bluff Mountain Creek is about equal to or slightly greater in size than the named portion of Bluff Mountain Creek; was about $3.5 \mathrm{~km}$ in length; and appeared to have similar habitat as Bluff Mountain Creek. In its upper reaches, this unnamed tributary is very steep with many short waterfalls. It cascades over bedrock outcroppings which would be barriers to fish dispersal. Some large, deep pools associated with bedrock and boulder dams in the stream channel are present in the headwaters, but these are scarce. Suitable fish habitat appeared to extend from its mouth up about $2.5 \mathrm{~km}$. In this portion the channel had a lower gradient and less confined valley bottom. Conifers, mainly lodge pole pine, dominate the riparian community for the stream's entire length. Overall, stream habitat was very similar to that of Bluff Mountain Creek, except for having slightly higher quality pools and slightly greater stream flow. No barriers to fish dispersal were located below $\mathrm{km} \mathrm{2.5,} \mathrm{although,} \mathrm{like} \mathrm{Bluff}$ Mountain Creek, many potential barrier placement sites are present. A portion of the headwaters has been logged, but harvest occurred well away from the stream channel. Again, there was no obvious reason why this stream would support brook trout when they are absent from so many other tributaries to the South Fork. Another small, unnamed stream entered this unnamed tributary to Bluff Mountain Creek from the east at km 1.2; however, this stream was too small to support fish.

Bluff Mountain Creek supported WCT up to stream kilometer 4.8, while both rainbow and brook trout were found at kilometer 1.6. Bluff Mountain Creek was the only stream sampled in the upper South Fork basin where brook trout were found during this study (Figures 1,2 and 6). A genetic sample of five WCT collected in 1997 indicated the WCT may be genetically pure, but 
was equivocal (Tews et al. 2000). Fin clips from 15 WCT from Bluff Mountain Creek and 8 WCT from its major tributary were collected for genetic analysis in 2000. Mottled sculpin were present in lower Bluff Mountain Creek.

Densities of WCT declined in an upstream direction from over 30 WCT $75 \mathrm{~mm}$ and longer per $100 \mathrm{~m}$ of stream length at $\mathrm{km} 0.8$ to about 20 per $100 \mathrm{~m}$ at $\mathrm{km} 4.0$ (Figure 6). A $100 \mathrm{~m}$ sample section located at stream kilometer $(\mathrm{km}) 1.6$ supported an estimated 4 (SE not calculated due to all fish captured on pass 1), 17 (SE: 1.2), and 17 (SE: 0.3) westslope cutthroat trout $<75 \mathrm{~mm}$, 75-149 $\mathrm{mm}$, and $150 \mathrm{~mm}$ and longer, respectively (Table 1 and Appendix B). The major unnamed tributary to Bluff Mountain Creek supported brook trout in its lower reaches and WCT

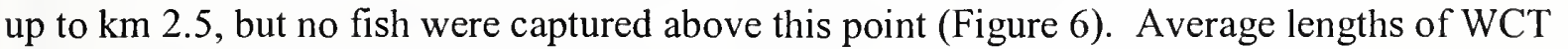
ranged from 121 to $166 \mathrm{~mm}$ in the sections where they were captured in Bluff Mountain Creek and its major unnamed tributary (Table 2). Length ranges for brook and rainbow trout illustrated that no juveniles $(<145 \mathrm{~mm})$ were captured, indicating that these species may not be reproducing in this stream system (Table 2). Conversely, a wide size range of WCT was captured (Figure 7).

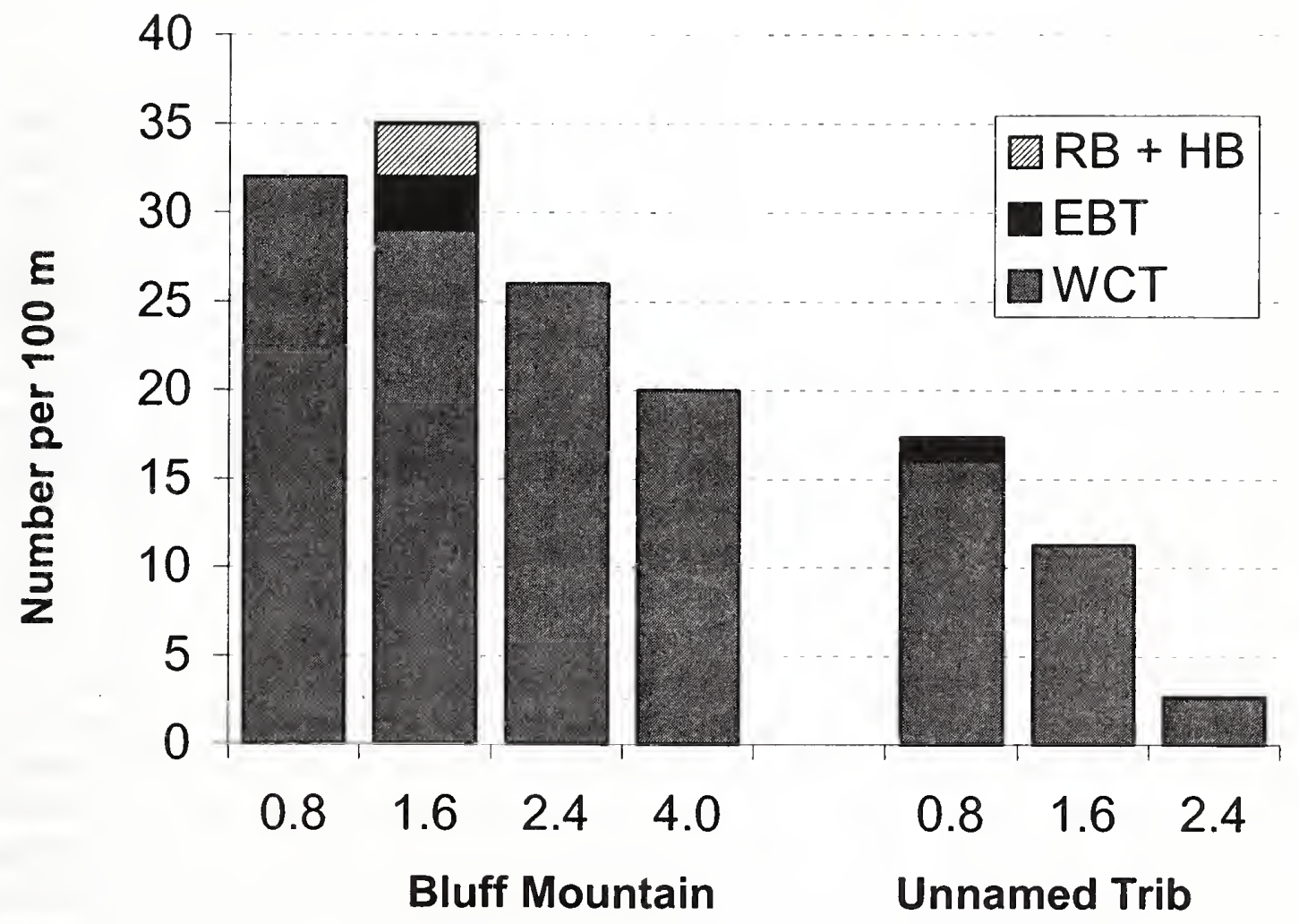

Figure 6. Relative abundance (number of fish $75 \mathrm{~mm}$ and longer captured on the first electrofishing pass per $100 \mathrm{~m}$ of stream length) for westslope cutthroat (WCT), brook (EBT), and rainbow and rainbow/cutthroat trout hybrids $(\mathrm{RB}+\mathrm{HB})$ in four sections of Bluff Mountain Creek and three sections of an unnamed tributary by stream kilometer. 


\section{Bluff Mountain}

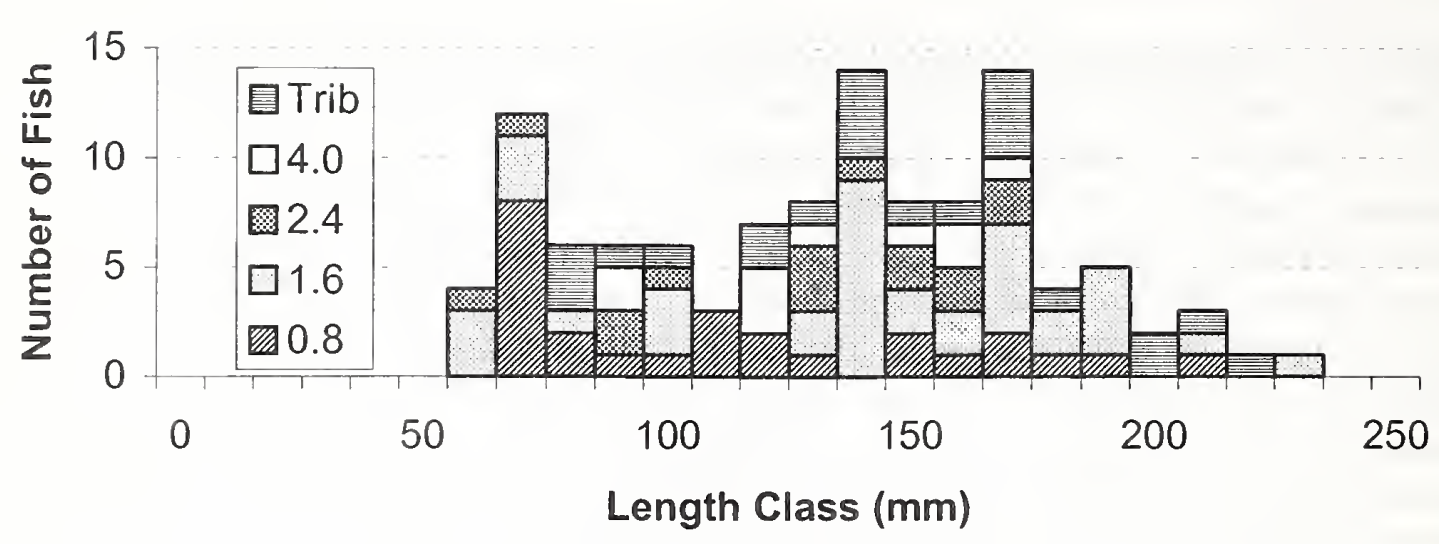

Figure 7. Length frequencies for westslope cutthroat trout in Bluff Mountain Creek (by stream kilometer up from mouth) and in three sections in a major unnamed tributary (TRIB) during July 2000.

A habitat survey conducted at $\mathrm{km} 1.6$ found that the streambed in Bluff Mountain Creek at this location was comprised primarily of cobbles and large gravels with sand and silt making up only about $10 \%$ of the streambed's surface (Table 3). Small and large woody debris were relatively scarce in the stream channel with only $33 \%$ of the large debris crossing the entire wetted channel (Table 3). Spawning habitat appeared to be relatively abundant and was likely not limiting fish recruitment (Table 3). Pool habitats made up $40 \%$ by number and slightly over $10 \%$ by length of all habitat types (Table 4). Wetted width averaged $2.4 \mathrm{~m}$ and depth averaged $19 \mathrm{~cm}$ (Table 4). Pool volumes averaged about $1.6 \mathrm{~m}^{3}$. Pools had an average maximum depth of $22 \mathrm{~cm}$. Instream cover and pool quality were ranked relatively low, while bank cover and stability were ranked as moderately high (Table 5).

\section{Cabin Creek}

Cabin Creek is a $6.5 \mathrm{~km}$ stream that enters the South Fork Judith at km 17.2 from the south. Cabin Creek has a moderate channel gradient and is confined by a relatively narrow valley bottom forested by dense conifers along its entire length. It has several extensive clear-cuts in its headwaters that appeared to be about 15-30 years old, based on regeneration of small conifers. A small, unnamed tributary enters Cabin Creek from the east at stream kilometer 4.0. No upstream fish movement barriers were observed in Cabin Creek. Fish distribution and relative abundance was assessed at stream kilometers $0.8,1.6,2.4,3.2,4.0,4.8,5.6$ and 6.4 fish population estimate and habitat surveys were conducted at $\mathrm{km} \mathrm{3.2}$. Average water temperatures in lower Cabin Creek remained below $13 \mathrm{C}$, while maximum water temperatures remained below $15 \mathrm{C}$ during the summer of 2000 (Figure 8).

Cabin Creek supported WCT up to $\mathrm{km} 6.4$; however, it appeared that the lower $0.8 \mathrm{~km}$ portion of the stream had been invaded by rainbow trout and several of the WCT captured at $\mathrm{km} 0.8$ 


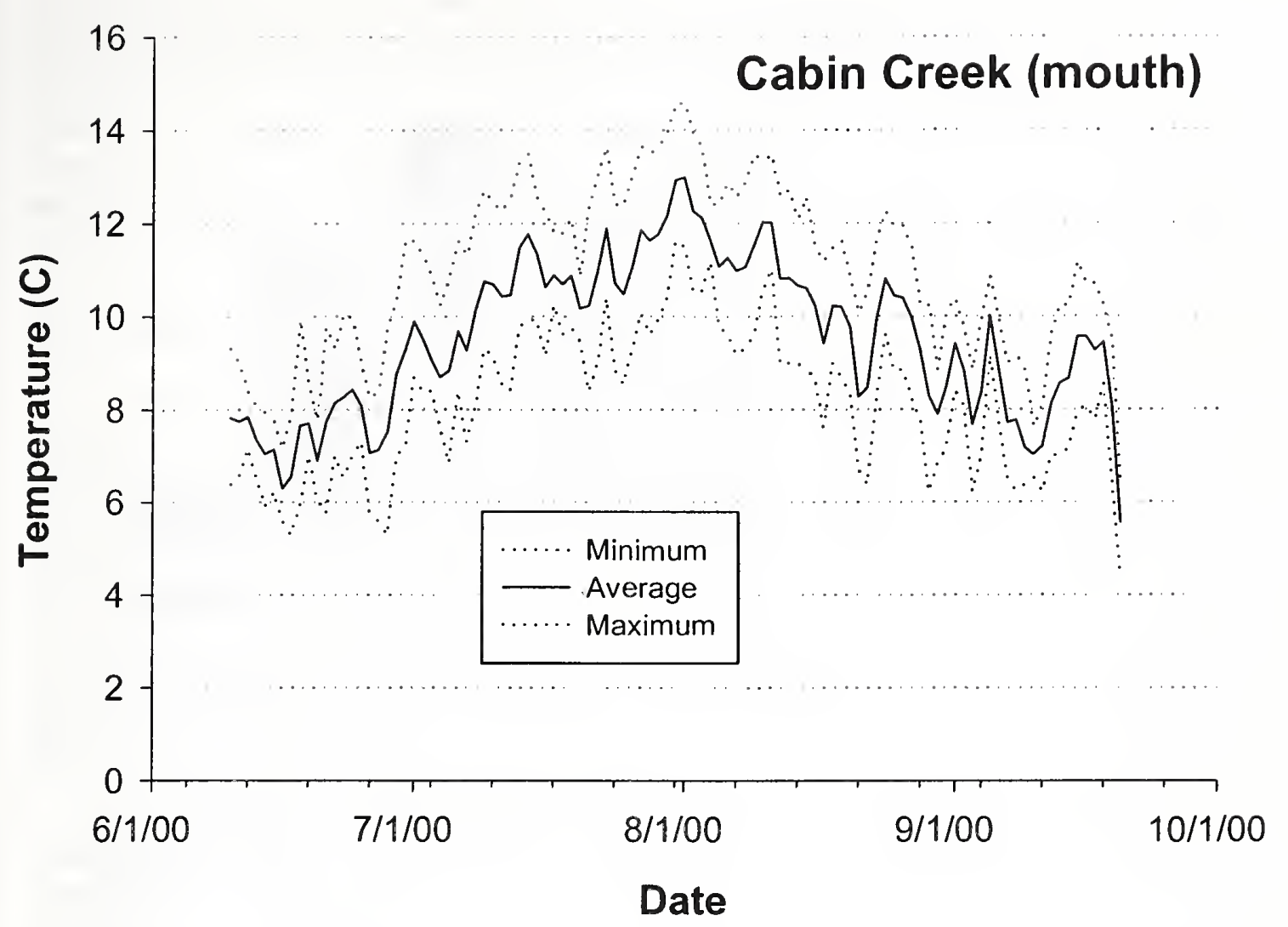

Figure 8. Average, minimum, and maximum water temperatures in Cabin Creek just above its mouth during the summer of 2000 .

appeared to be WCT/rainbow hybrids. A genetic sample of 5 fish collected in 1997 indicated that these fish had alleles characteristic of both WCT and Yellowstone cutthroat trout based on allozyme analysis (Tews et al. 2000). Fins from 25 fish from throughout Cabin Creek were obtained in 2000 for further genetic analysis. Densities of WCT were highest in middle portions of the creek (km 3.2 to 5.6), with over 40 WCT $75 \mathrm{~mm}$ and longer per $100 \mathrm{~m}$ of stream length compared to 20 or less per $100 \mathrm{~m}$ in other sample sites (Figure 9). A $75 \mathrm{~m}$ sample section located at stream $\mathrm{km} 3.2$ supported an estimated 28 (SE: 0.6) and 15 (SE not calculated due to all fish captured on pass 1) westslope cutthroat trout $75-149 \mathrm{~mm}$ and $150 \mathrm{~mm}$ and longer, respectively (Table 1 and Appendix B). No fish were observed in the unnamed tributary to Cabin Creek. Average lengths of WCT ranged from 124 to $174 \mathrm{~mm}$ in the sections where they were captured in Cabin Creek (Table 2). WCT ranged in length from 51 to $223 \mathrm{~mm}$, while the single fish identified as a rainbow trout was $192 \mathrm{~mm}$ long (Table 2 and Figure 10).

A habitat survey conducted at $\mathrm{km} 3.2$ found that the streambed in Cabin Creek at this location was comprised primarily of large and small gravels with sand and silt making up about $30 \%$ of the streambed's surface (Table 3 ). Small and large woody debris were relatively abundant in the stream channel with over two thirds of the large debris crossing the entire wetted channel (Table 3). Spawning habitat appeared to be relatively abundant and was likely not limiting fish 


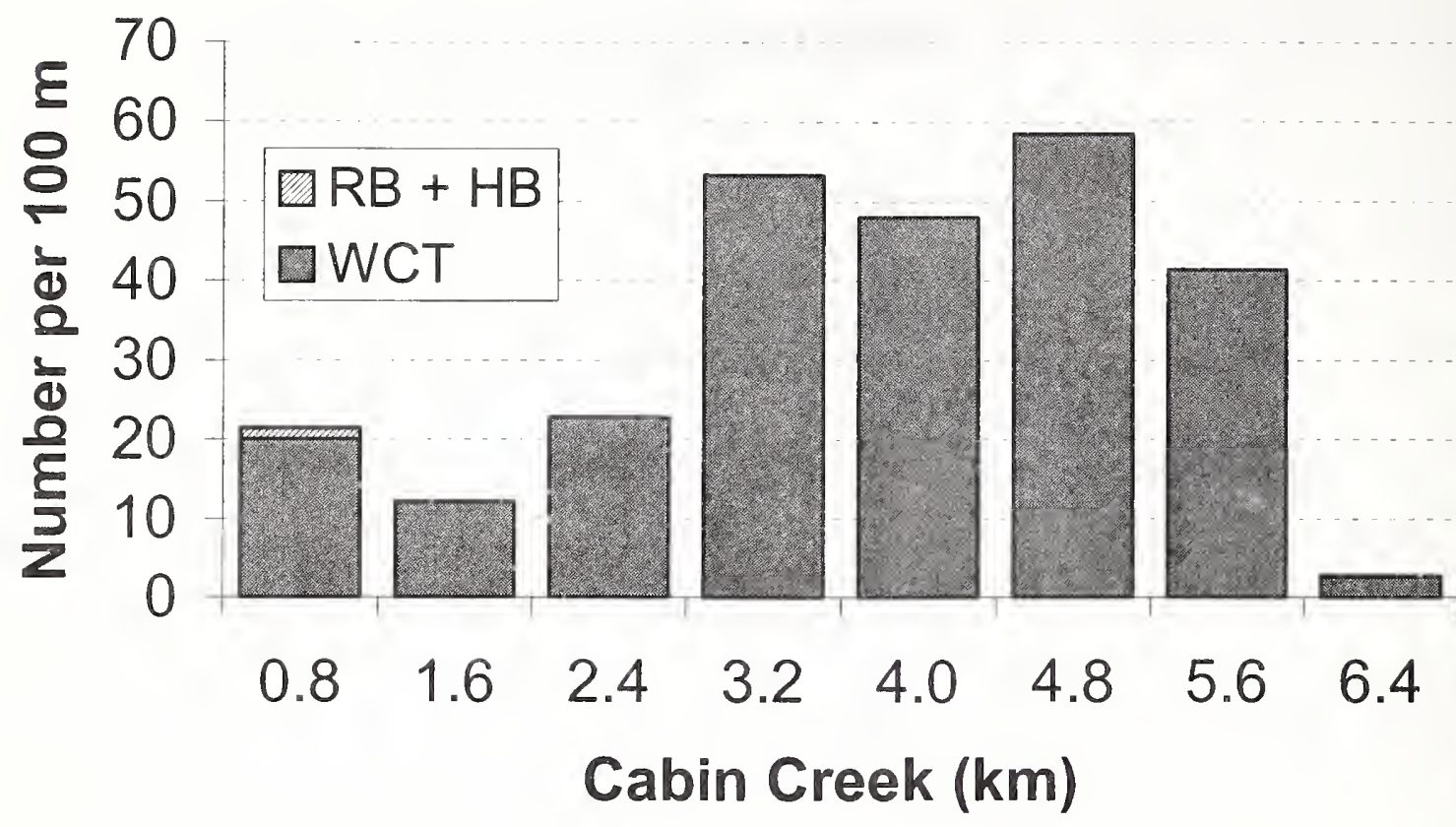

Figure 9. Relative abundance (number of fish $75 \mathrm{~mm}$ and longer captured on the first electrofishing pass per $100 \mathrm{~m}$ of stream length) for westslope cutthroat (WCT) and rainbow and cutthroat/rainbow trout hybrids $(\mathrm{RB}+\mathrm{HB})$ in eight sections of Cabin Creek by stream kilometer.

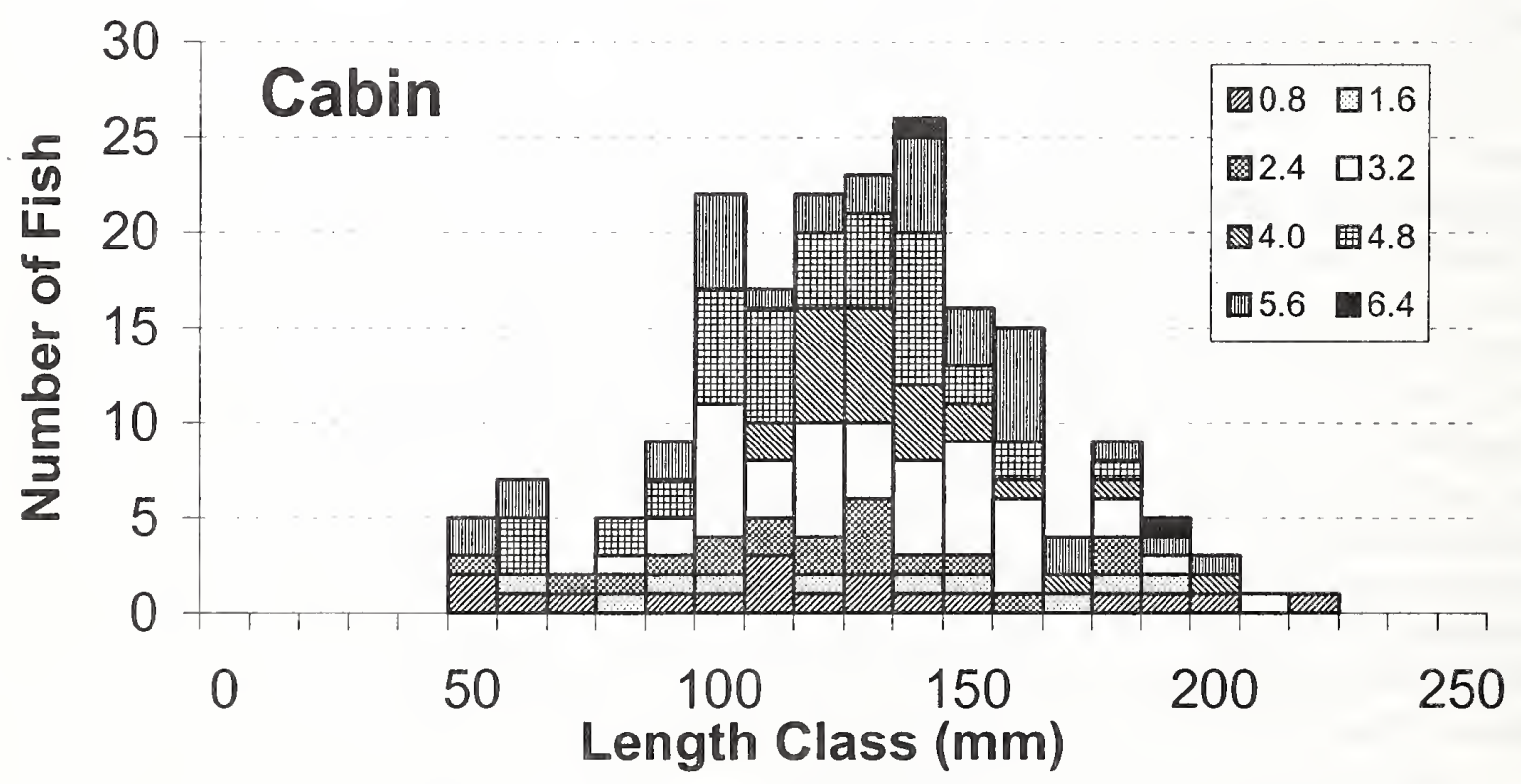

Figure 10. Length frequencies for westslope cutthroat trout in Cabin Creek (by stream kilometer up from mouth) during late June ( $\mathrm{km} 1.6$ and 3.2) and late July (km 0.8, 2.4, and 4.0 to 6.4) 2000 . 
recruitment; however, high levels of fine sediments might be impacting embryo survival (Table 3). Pool habitats made up $50 \%$ by number and slightly over $25 \%$ by length of all habitat types (Table 4). Wetted width averaged $2.1 \mathrm{~m}$ and depth averaged $16.5 \mathrm{~cm}$ (Table 4). Pool volumes averaged about $1.4 \mathrm{~m}^{3}$. Pools had an average maximum depth of $23 \mathrm{~cm}$. Instream and bank cover, bank stability, and pool quality were ranked as moderately high (Table 5). Undercut banks provided much of the bank cover. Light riparian use by wildlife was observed in this portion of the stream.

\section{Cross Creek}

Cross Creek is a 3.3 kilometer long stream that enters the South Fork Judith River from the south at $\mathrm{km} \mathrm{20.1.} \mathrm{A} \mathrm{barrier} \mathrm{to} \mathrm{upstream} \mathrm{fish} \mathrm{movement} \mathrm{was} \mathrm{observed} \mathrm{near} \mathrm{stream} \mathrm{km} \mathrm{1.3.} \mathrm{No} \mathrm{fish}$ were observed above this barrier. Fish habitat below the fish barrier was classified as good with numerous pools. The stream below this barrier flowed through dense conifer forest and the channel gradient was moderate to low. Rockslides were observed at a few locations where the channel intersected bedrock outcrops. Bare talus slopes contributed sediment to the stream channel at these locations causing localized channel instability. An abandoned logging road crossed Cross Creek a short distance above its mouth and the culvert at this road crossing may inhibit fish passage upstream during some flows. The channel flows through a small clear-cut near its mouth. A depletion fish population estimate was made at $\mathrm{km} 0.8$.

Cross Creek supported WCT up to stream kilometer 1.3, where the fish barrier was located. A genetic sample of five WCT collected in 1997 indicated the WCT may be genetically pure, but was equivocal (Tews et al. 2000). Fin clips from 24 WCT from Cross Creek were collected for genetic analysis in 2000. Densities of WCT were moderately high (34 WCT $75 \mathrm{~mm}$ and longer per $100 \mathrm{~m}$ of stream) when compared to other streams. A $90 \mathrm{~m}$ sample section located at stream km 0.8 supported an estimated 24 (SE: 0.4) and 10 (SE: 0.3 ) WCT 75-149 mm and $150 \mathrm{~mm}$ and longer, respectively (Table 1 and Appendix B). The average length of captured WCT was 134 $\mathrm{mm}$ and ranged from 59 to $188 \mathrm{~mm}$ (Table 2 and Figure 11).

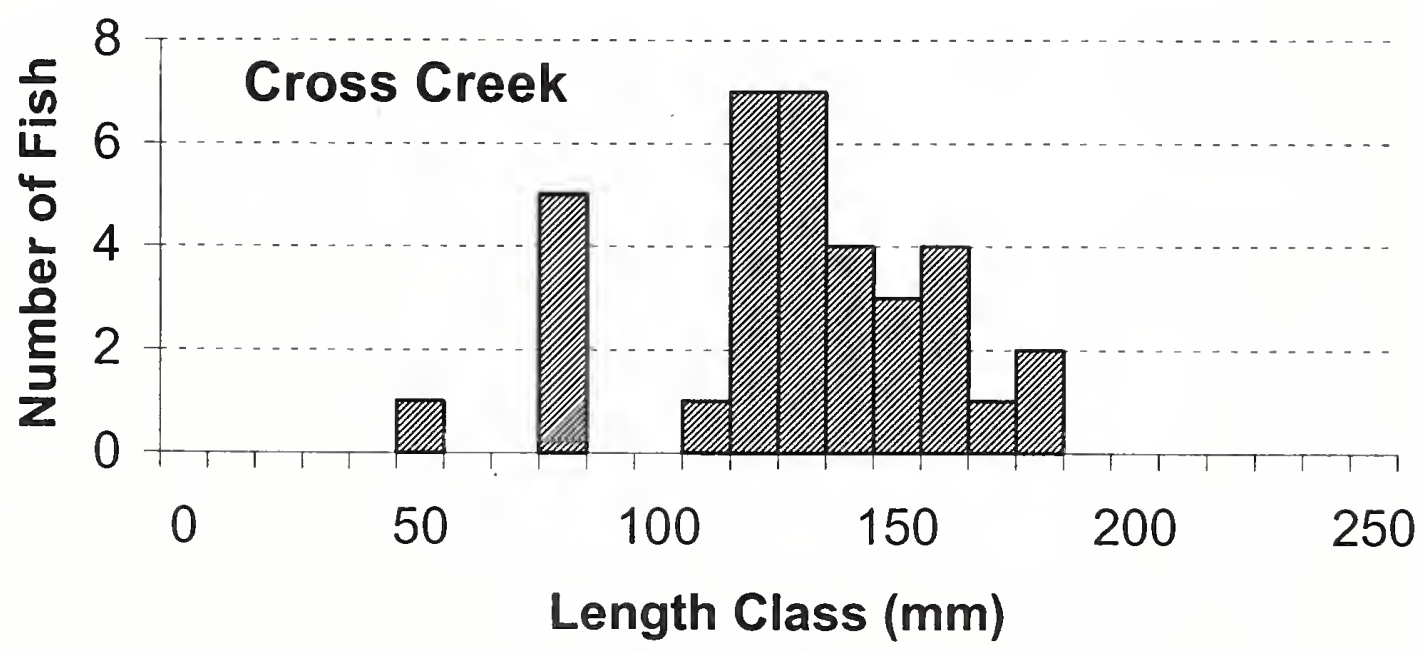

Figure 11. Length frequencies for westslope cutthroat trout in Cross Creek at stream kilometer 0.8 during late July 2000. 
A habitat survey conducted at $\mathrm{km} .08$ found that the streambed in Cross Creek at this location was comprised primarily of small gravels with sand and silt making up about $20 \%$ of the streambed's surface (Table 3). Woody debris was relatively abundant in the stream channel and much more of this debris was large, although relatively few debris pieces crossed the entire wetted channel (Table 3). Spawning habitat appeared to be relatively abundant and was likely not limiting fish recruitment (Table 3). Pool habitats made up $44 \%$ by number and about $29 \%$ by length of all habitat types (Table 4). Wetted width averaged $2.2 \mathrm{~m}$ and depth averaged $11 \mathrm{~cm}$ (Table 4). Pool volumes averaged about $1.1 \mathrm{~m}^{3}$. Bank stability and pool quality were ranked relatively high (Table 5).

\section{Deadhorse Creek}

Deadhorse Creek is approximately $6.5 \mathrm{~km}$ and enters the South Fork Judith River from the south at about river kilometer 21.8. The creek's headwaters flow through a relatively low gradient bench for about $2.5 \mathrm{~km}$ where extensive logging has occurred in the past; however, most timber harvest units were located away from the stream channel. At about stream $\mathrm{km} 4.0$ the stream enters a relatively confined valley with a dense conifer forest along the stream and higher channel gradient to the stream's mouth at the South Fork. No upstream fish migration barriers were found. Fish habitat was generally fair to good; however, high levels of fine sediment may impact spawning habitats. Fish distribution and relative abundance was assessed at stream kilometers $0.8,1.6,2.4,3.2,4.0$, and 4.8 , and fish population estimate and habitat surveys were conducted at km 3.2. A small tributary enters Deadhorse Creek from the west at about stream $\mathrm{km} 4.1$ and while fish were observed in this small tributary it was not sampled. Average water temperatures in lower Deadhorse Creek remained below $14 \mathrm{C}$, while maximum water temperatures climbed to over $16 \mathrm{C}$ during the summer of 2000 (Figure 12).

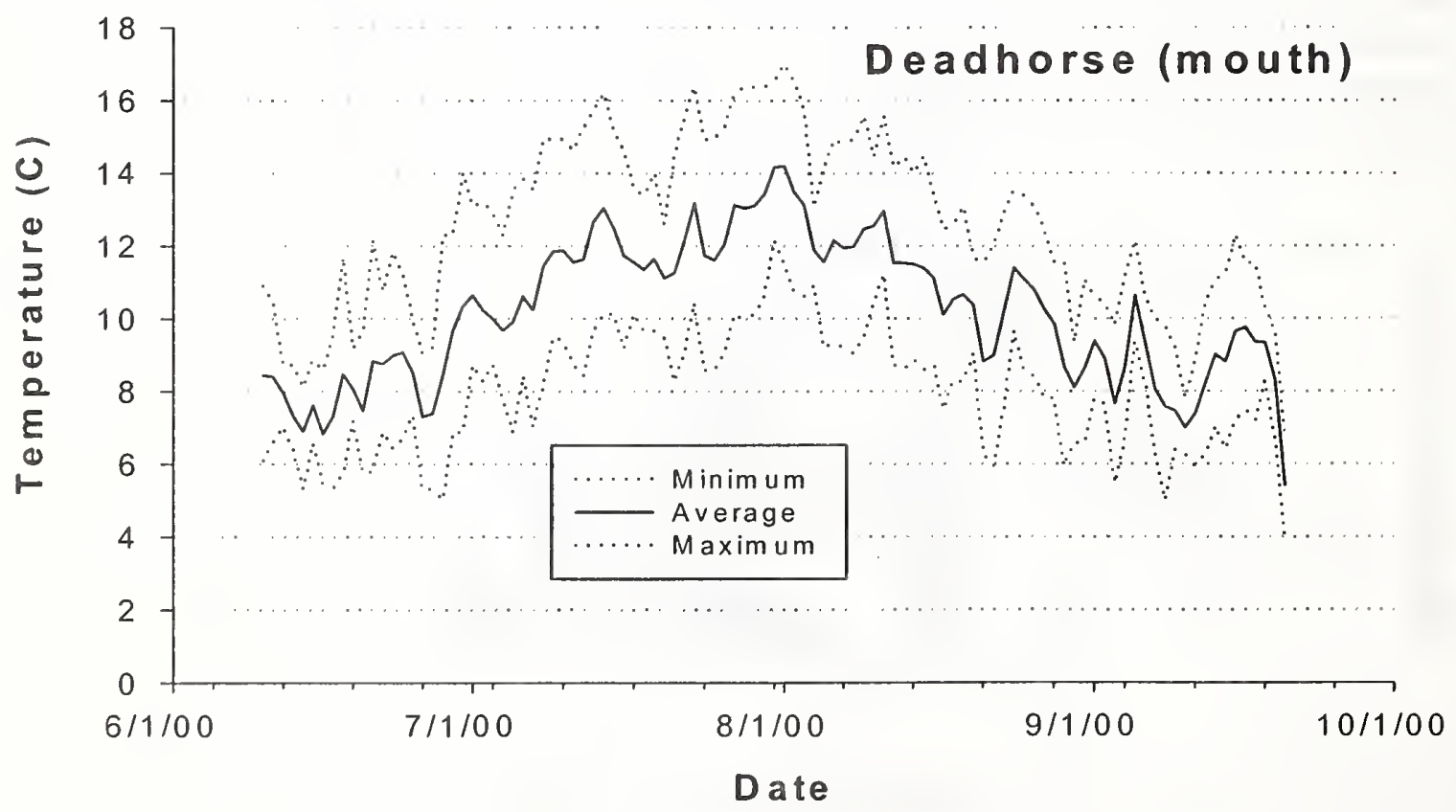

Figure 12. Average, minimum, and maximum water temperatures in Deadhorse Creek just above its mouth during the summer of 2000. 
Deadhorse Creek supported only WCT. A genetic sample of 5 fish collected from the upper reach of the stream in 1997 indicated that these fish had alleles characteristic of only WCT, but these results were equivocal (Tews et al. 2000). Fins from 25 fish from throughout Deadhorse Creek were obtained in 2000 for further genetic analysis. Densities of WCT were highest in middle portions of the creek ( $\mathrm{km} 2.4$ to 4.0), with over 25 WCT $75 \mathrm{~mm}$ and longer per $100 \mathrm{~m}$ of stream length compared to 15 or less per $100 \mathrm{~m}$ in other sample sites (Figure 13). A $75 \mathrm{~m}$ sample section located at stream $\mathrm{km} 3.2$ supported an estimated 16 (SE: 3.9) and 13 (SE: 0.6) westslope cutthroat trout 75-149 $\mathrm{mm}$ and $150 \mathrm{~mm}$ and longer, respectively (Table 1 and Appendix B). As stated above, fish were observed in the unnamed tributary to Deadhorse Creek, but this stream was not sampled. Average lengths of WCT ranged from 83 to $160 \mathrm{~mm}$ with fish of larger average lengths captured in the lower portions of the stream and of smaller average length captured in the upper portions (Table 2). WCT ranged in length from 56 to $226 \mathrm{~mm}$ (Table 2 and Figure 14).

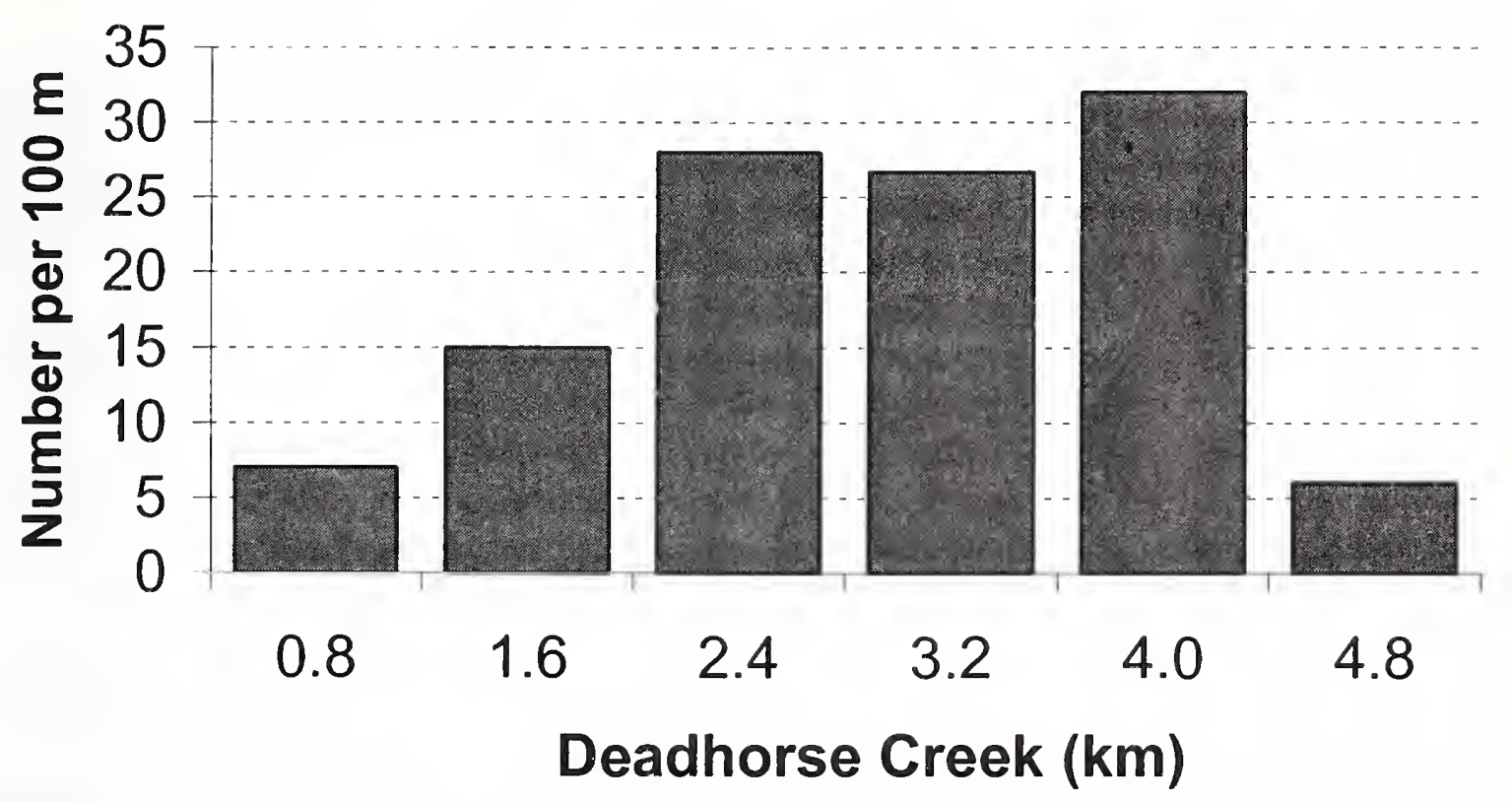

Figure 13. Relative abundance (number of fish $75 \mathrm{~mm}$ and longer captured on the first electrofishing pass per $100 \mathrm{~m}$ of stream length) for westslope cutthroat trout in six sections of Deadhorse Creek by stream kilometer.

A habitat survey conducted at km 3.2 found that the streambed in Deadhorse Creek at this location was comprised primarily of large gravels and cobbles with sand and silt making up about $25 \%$ of the streambed's surface (Table 3 ). Small woody debris was relatively scarce, but large woody debris was abundant in the stream channel (Table 3). About $37 \%$ of the large debris crossed the entire wetted channel (Table 3). Spawning habitat appeared to be limited and limiting fish recruitment (Table 3 ). Pool habitats made up $50 \%$ by number and about $35 \%$ by length of all habitat types (Table 4). Wetted width averaged $1.7 \mathrm{~m}$ and depth averaged $21 \mathrm{~cm}$ (Table 4). Pool volumes averaged about $2.1 \mathrm{~m}^{3}$. Pools had an average maximum depth of 29 $\mathrm{cm}$. Instream and bank cover, bank stability, and pool quality were all ranked as moderately high, while riparian use was low (Table 5). 


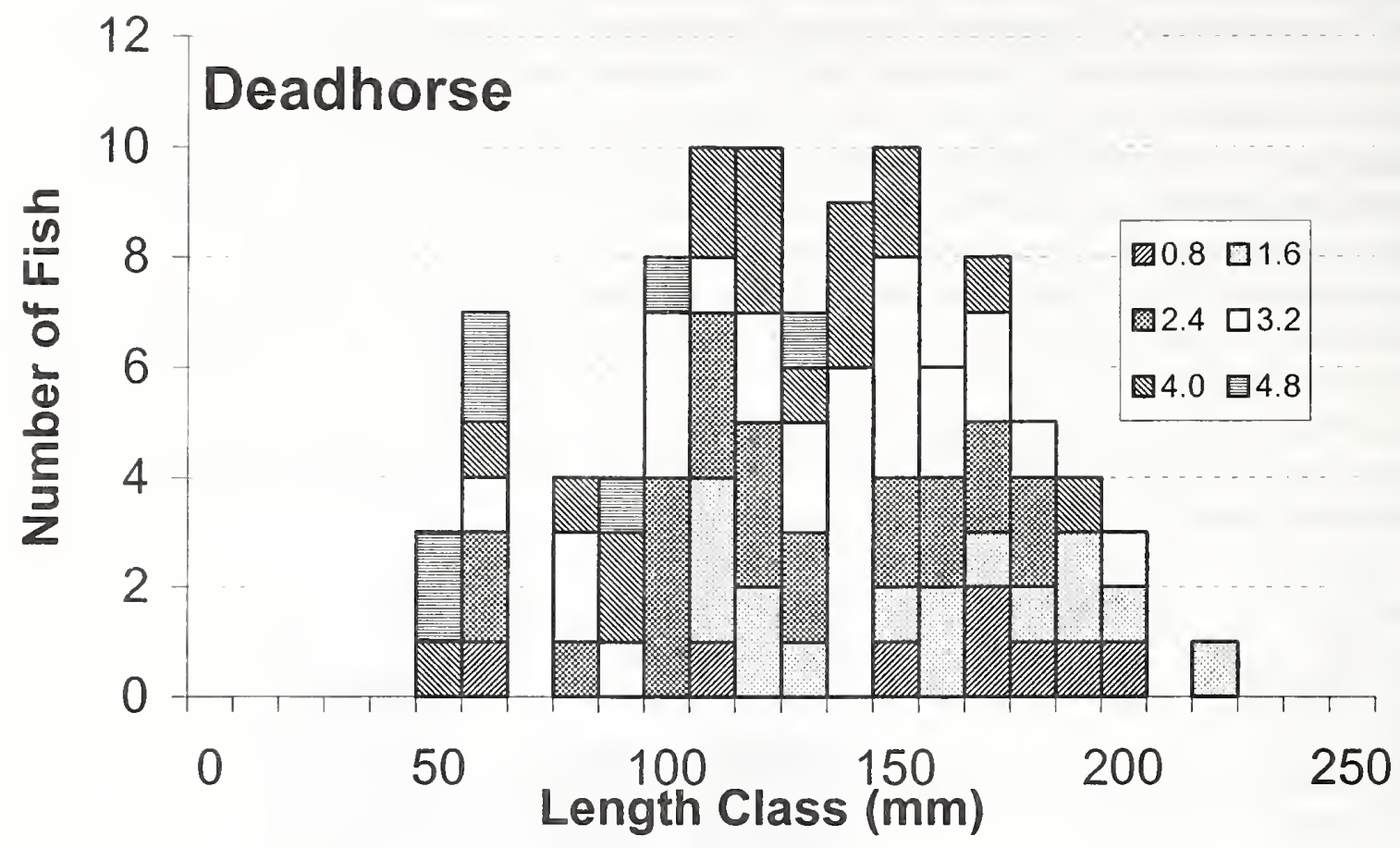

Figure 14. Length frequencies for westslope cutthroat trout in six sections of Deadhorse Creek (by stream kilometer) during late July 2000.

\section{Russian Creek}

Russian Creek is approximately $4.6 \mathrm{~km}$ in length and enters the South Fork Judith River from the north at about river km 23.6 (Figure 1). A large unnamed tributary enters Russian Creek from the west at about km 2.7, or about $200 \mathrm{~m}$ above the South Fork Road, and this tributary contributes most of the flow to Russian Creek. About $200 \mathrm{~m}$ above the junction of this unnamed tributary Russian Creek did not flow and did not even have a very defined stream channel. A large beaver dam complex surrounded by small willows and sparse conifers impounds both Russian Creek and the unnamed tributary immediately above the South Fork Road. While this beaver complex supported some fish, much of it was too shallow to provide much fish habitat.

The unnamed tributary was about $2.4 \mathrm{~km}$ in length. The upper portions of this tributary had a relatively high channel gradient, narrow valley bottom, and flowed through a conifer forest. An old clear-cut was adjacent to the stream channel near its headwaters and the stream channel below this area was entrenched and choked with woody debris. A fish migration barrier consisting of a steep cascade was observed in this unnamed tributary at about km 1.5. No fish were observed above this point and the habitat above this point was deemed marginal for fish. From this barrier downstream the channel gradient moderated and the valley bottom was wider. Willows dominated the riparian community in the lower portion of this unnamed tributary. Fish habitat was considered moderate with some channel instability observed. Fish distribution and 
relative abundance was assessed at stream kilometers 0.4 and 0.8 and fish population estimate and habitat surveys were conducted at $\mathrm{km} 0.4$.

The culvert at the South Fork Road crossing of Russian Creek was believed to be a barrier to upstream fish movement, a conclusion also reached by Tews et al. (2000). The channel gradient above the South Fork Road was relatively low and the valley was generally unconfined above the road. Below the South Fork Road, Russian Creek enters a more confined valley, channel gradient increases, and conifers dominate the riparian community. Fish distribution and relative abundance was assessed at stream kilometers 0.8 and 2.4 , but no fish population estimate or habitat surveys were conducted. Water temperatures were very high in Russian Creek immediately below the road crossing, probably a result of warming of the water in the shallow, broad beaver complex located just above the road (Figure 15; top graph). Water temperatures were much lower near the mouth of Russian Creek, probably due to inflows of groundwater in the portion of the stream from the road to its mouth (Figure 15; bottom graph).

Westslope cutthroat trout was the only fish species captured or seen in Russian Creek and its major tributary. Five fish were collected from both the lower and upper portions of Russian Creek for genetic analyses in 1996 (Tews et al. 2000). The fish from upper Russian Creek had alleles characteristic of only WCT, while the fish from lower Russian Creek had alleles characteristic of both WCT and rainbow trout. Ten fin clips were obtained from one site in the unnamed tributary to Russian Creek and ten fin clips were taken from two sites in Russian Creek for PINE genetic testing. These genetic tests have not yet been completed.

Densities of WCT were highest at km 2.4 in Russian Creek, with nearly 35 WCT $75 \mathrm{~mm}$ and longer per $100 \mathrm{~m}$ of stream length; moderate in the two sections within the major unnamed tributary (15 to 25 per $100 \mathrm{~m}$ ); and relatively low in the lower portion of Russian Creek (Figure 16). A $100 \mathrm{~m}$ sample section located at stream $\mathrm{km} 0.4$ in the unnamed tributary supported an estimated 5 (SE: 1.2), 18 (SE: 0.8), and 10 (SE: 0.7) westslope cutthroat trout <75 mm, 75-149 $\mathrm{mm}$, and $150 \mathrm{~mm}$ and longer, respectively (Table 1 and Appendix B). Average lengths of WCT were 129 and $127 \mathrm{~mm}$ in the two Russian Creek sections and 117 and $147 \mathrm{~mm}$ in the unnamed tributary (Table 2). WCT ranged in length from 57 to $245 \mathrm{~mm}$ (Table 2 and Figure 17).

A habitat survey conducted at $\mathrm{km} 0.4$ of the major unnamed tributary found that the streambed at this location was comprised primarily of small gravels with sand and silt making up about $25 \%$ of the streambed's surface (Table 3). Small and large woody debris was relatively scarce with about 14 of the small and $44 \%$ of the large debris crossing the wetted channel (Table 3 ). Spawning habitat appeared to be very abundant (Table 3). Pool habitats made up $40 \%$ by number and about $21 \%$ by length of all habitat types (Table 4 ). Wetted width averaged $1.1 \mathrm{~m}$ and depth averaged $12 \mathrm{~cm}$ (Table 4). Pool volumes averaged about $0.7 \mathrm{~m} 3$ with residual pool volumes estimated as $0.2 \mathrm{~m} 3$. Pools had an average maximum depth of $16 \mathrm{~cm}$. Instream and bank cover were moderately high, while bank stability, pool quality, and riparian use were moderate to low (Table 5). Woody debris and aquatic vegetation made up instream cover. While there were many pools, they were small and of relatively low quality. Several observed raw and eroding banks led to a moderate bank stability rating; however, most banks that were not actively eroding had relatively good vegetative cover. Human recreational use and wildlife and livestock grazing and browsing were impacting riparian areas. 

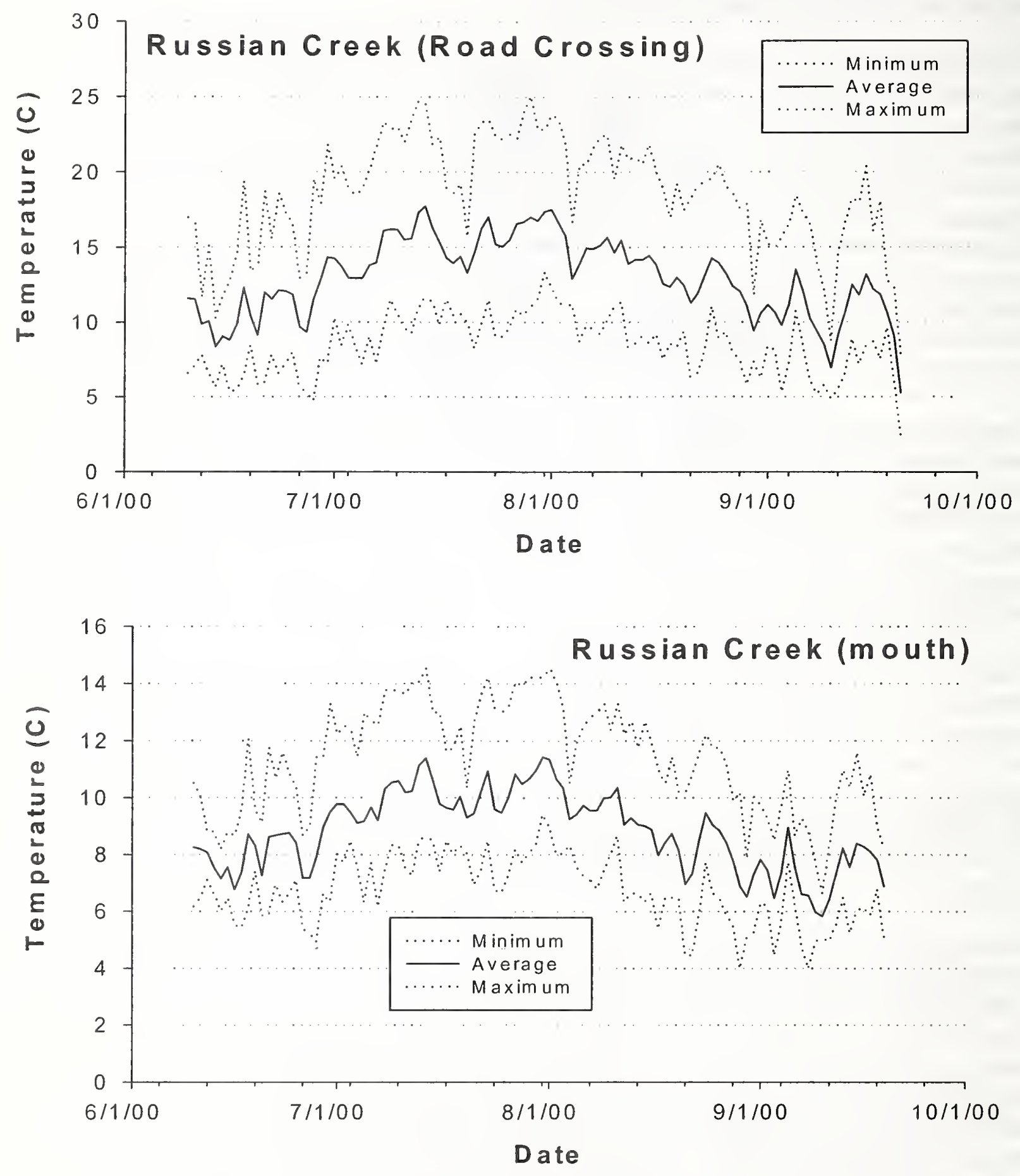

Figure 15. Average, minimum, and maximum water temperatures in Russian Creek just below the South Fork Road crossing (top) and just above its mouth (bottom) during the summer of 2000 . 


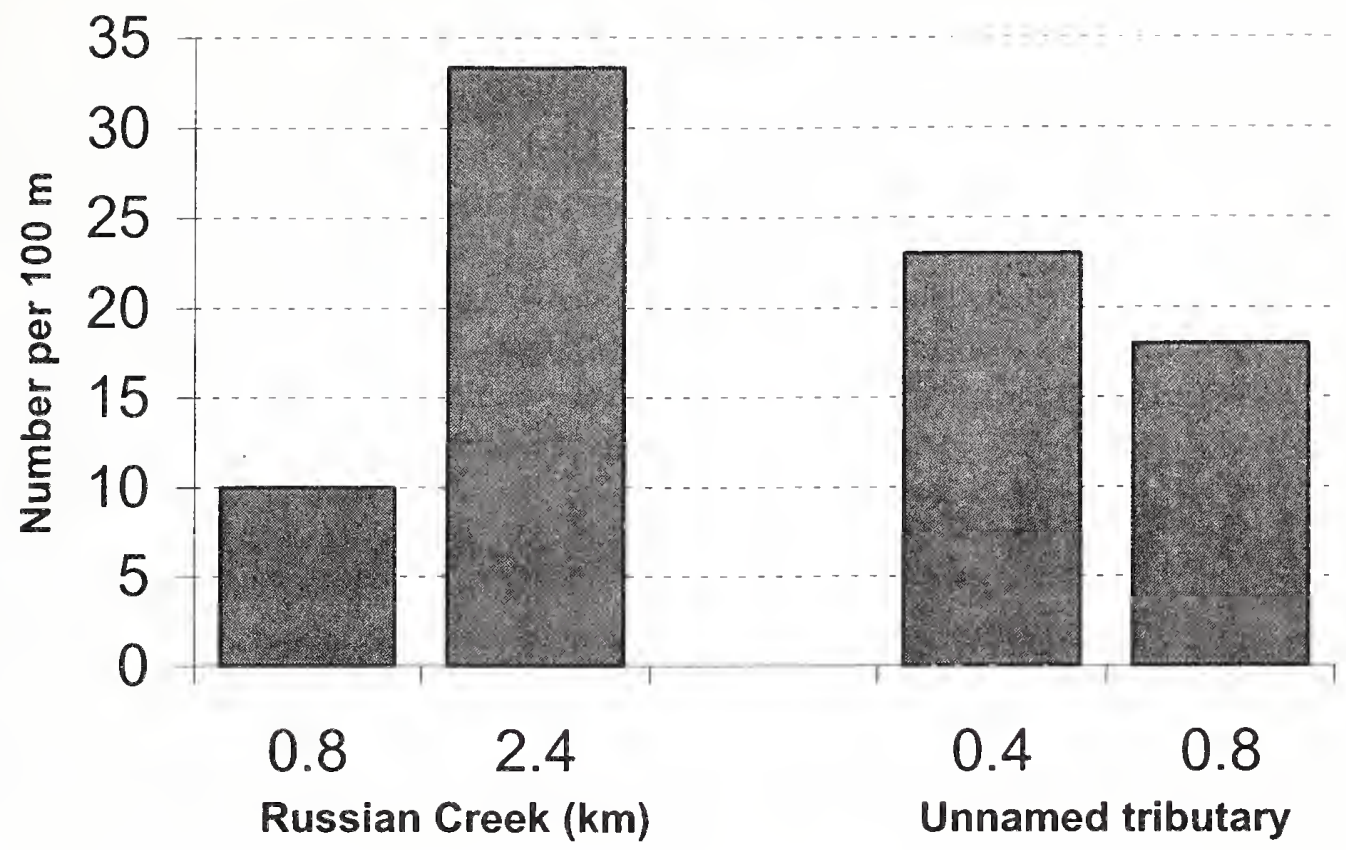

Figure 16. Relative abundance (number of fish $75 \mathrm{~mm}$ and longer captured on the first electrofishing pass per $100 \mathrm{~m}$ of stream length) for westslope cutthroat trout during 2000 in two sections of Russian Creek (left) and an unnamed tributary to Russian Creek (right) by stream kilometer.

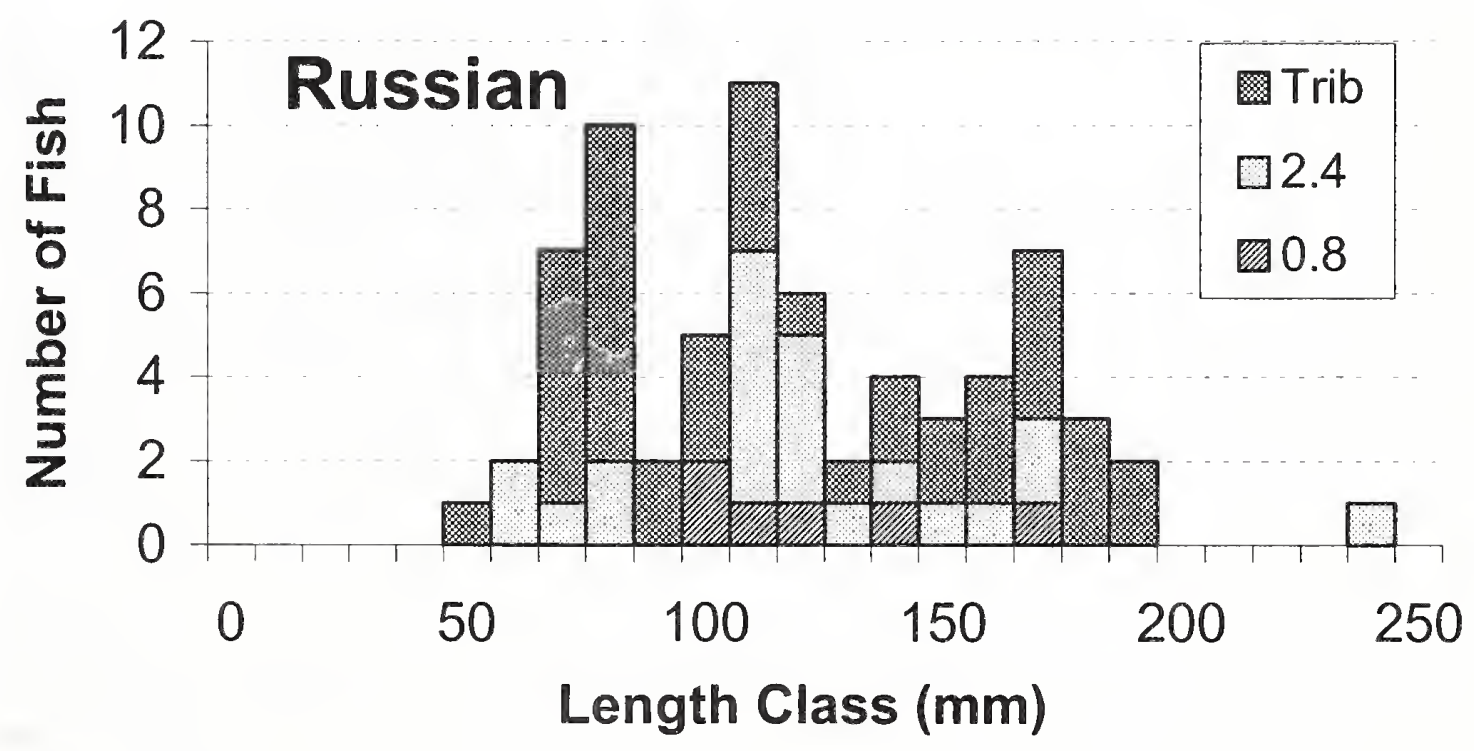

Figure 17. Length frequencies for westslope cut throat trout in two sections of Russian Creek (by stream kilometer) and its major unnamed tributary (Trib) during late June 2000. 


\section{South Fork Judith River}

The upper portion of the South Fork Judith River was sampled from river km 19.0 up river. From the mouth of an unnamed spring at river km 29.2 the South Fork's channel is relatively undefined and flows appear too low to support fish. From this unnamed spring down to Big Hill Creek (river $\mathrm{km} \mathrm{27.6)} \mathrm{the} \mathrm{valley} \mathrm{is} \mathrm{unconfined,} \mathrm{channel} \mathrm{gradient} \mathrm{is} \mathrm{low,} \mathrm{and} \mathrm{riparian} \mathrm{vegetation}$ consisted mostly of grasses, sedges, and forbs with occasional conifers and willows. Immediately below the mouth of Big Hill Creek, the South Fork enters a large beaver complex that extends downstream several hundred meters. The South Fork is a relatively small stream until the flows of Russian and Deadhorse creeks enter it, where it triples its flow and begins to enter a narrower confined valley. Below Deadhorse Creek the South Fork's channel gradient increases, the stream's substrate becomes somewhat larger, and bedrock outcrops occasionally intersect the stream channel and form deep pools. Woody debris also becomes more abundant below Deadhorse Creek where the stream flows through dense coniferous forests interspersed with a few stringer meadows. Many high quality pools are formed by woody debris in this reach of the South Fork. Fish distribution and relative abundance was assessed at river kilometers $19.3,22.5,23.3,24.1,24.9,25.7,26.5,27.4$, and 28.3 and fish population estimate and habitat surveys were conducted at kilometers 19.3, 22.5, and 25.7.

Three thermographs recorded water temperatures in the South Fork (Figure 1). Average water temperatures below Bluff Mountain Creek reached $14 \mathrm{C}$, but were generally between 10 and 14 C, while maximum water temperatures exceeded $16 \mathrm{C}$ during the summer of 2000 (Figure 18; top graph). Average water temperatures in the South Fork immediately below the mouths of Big Hill and Corral creeks were generally 8 to $10 \mathrm{C}$ or 6 to $8 \mathrm{C}$, respectively (Figure 18; lower two graphs). Maximum summer temperatures reached $17 \mathrm{C}$ below Big Hill and only $13 \mathrm{C}$ below Corral Creek.

Westslope cutthroat and rainbow trout were captured in the South Fork Judith River. Five fish were collected for genetic analyses from the South Fork Judith River above Russian Creek and below Deadhorse Creek in 1997 (Tews et al. 2000). The fish from above Russian Creek had only alleles characteristic of WCT based on allozyme testing, but the results were equivocal. The sample from below Deadhorse Creek could not be analyzed because the fish thawed. A sample of 30 fish from the South Fork above Russian Creek taken in 1984 indicated 98\% of diagnostic alleles were characteristic of WCT with $2 \%$ rainbow and Yellowstone cutthroat trout (Tews et al. 2000). Fin clips were obtained from 22 fish throughout the South Fork in 2000 for PINE genetic testing, but these genetic tests have not yet been completed. Two fish identified as rainbow trout were captured in the lowermost sampling section $(\mathrm{km} \mathrm{19.3)}$ and it is likely that some fish identified as WCT could have been hybrids between WCT and rainbow trout.

Densities of WCT were highest at km 26.5, with nearly 70 WCT $75 \mathrm{~mm}$ and longer per $100 \mathrm{~m}$ of stream length and moderately high in all remaining sample sections (over 40 WCT per $100 \mathrm{~m}$ ) except the two sections at $\mathrm{km} 24.1$ and 24.9 (densities from about 25 to 35 WCT per $100 \mathrm{~m}$ ) and the upper section at 28.3 (density about 12 WCT per 100 m; Figure 19). A 135 m sample section 

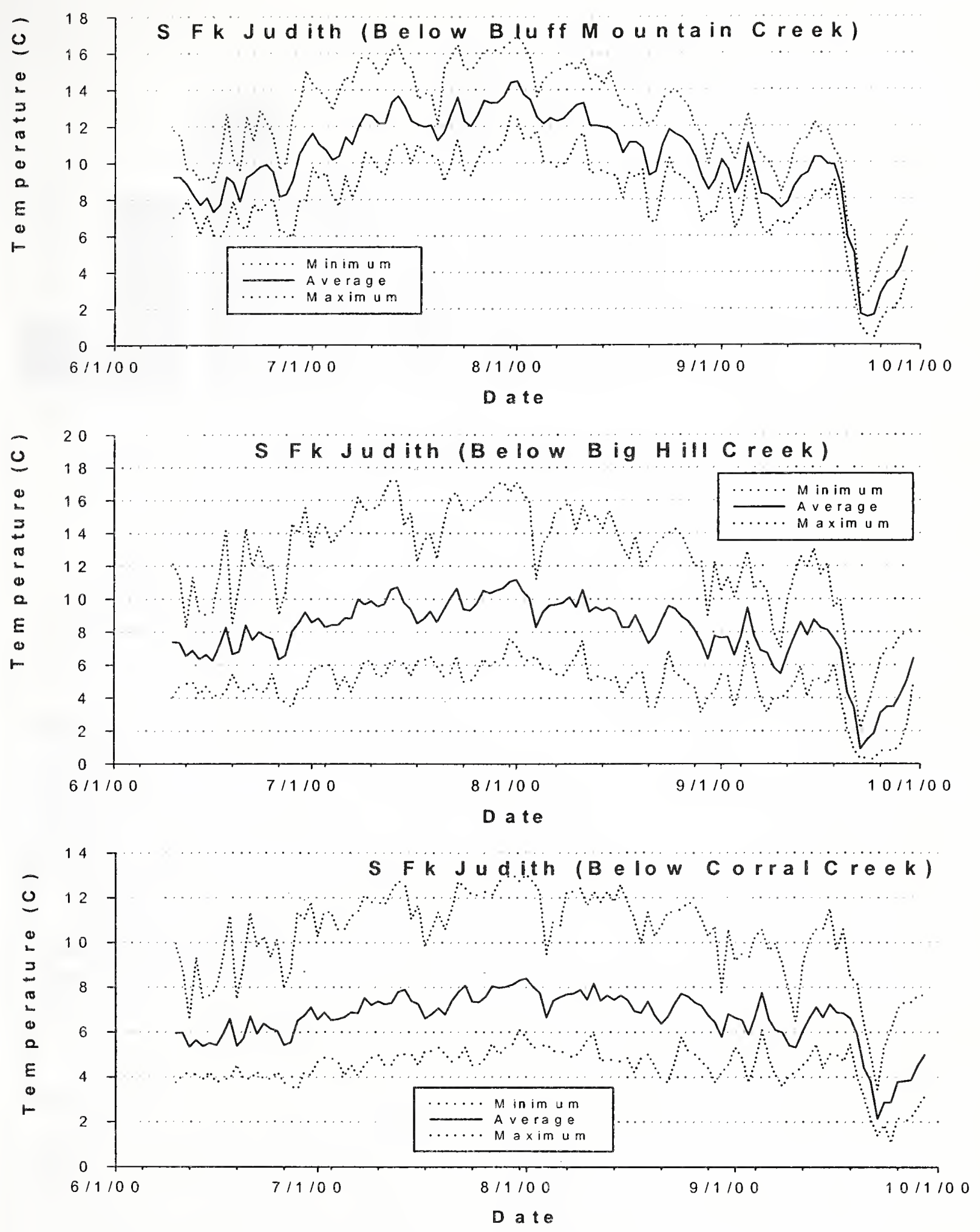

Figure 18. Average, minimum, and maximum water temperatures in the South Fork Judith River below the mouths of Bluff Mountain Creek (top), Big Hill Creek (middle), and Corral Creek (bottom) during the summer of 2000. 


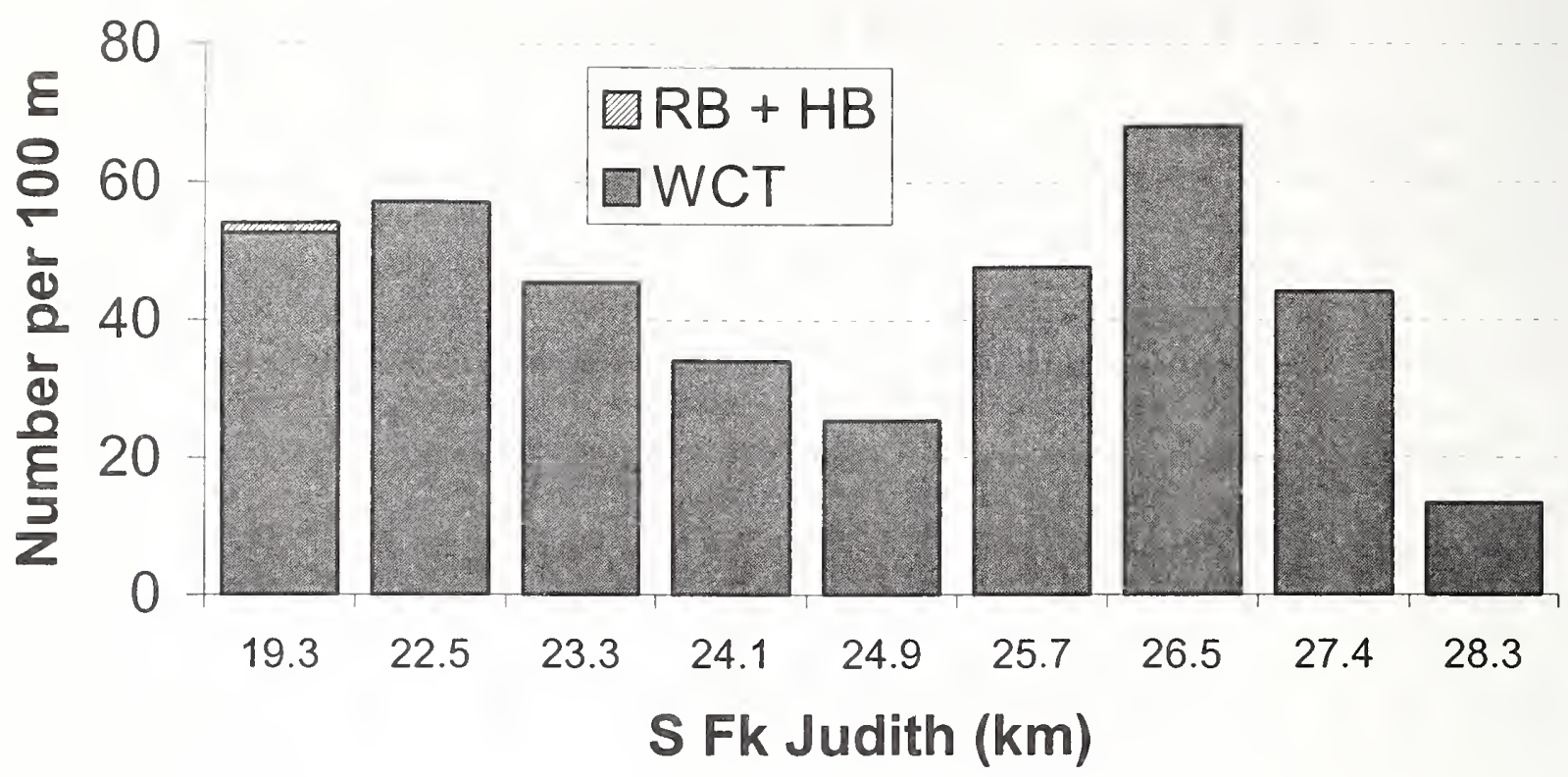

Figure 19. Relative abundance (number of fish $75 \mathrm{~mm}$ and longer captured on the first electrofishing pass per $100 \mathrm{~m}$ of stream length) for westslope cutthroat trout (WCT) and rainbow trout $(\mathrm{RB}+\mathrm{HB})$ during 2000 in nine sections of the South Fork Judith River by stream kilometer.

located at river $\mathrm{km} 19.3$ supported an estimated 26 (SE: 0.4) and 51 (SE: 0.6) westslope cutthroat trout $75-149 \mathrm{~mm}$ and $150 \mathrm{~mm}$ and longer, respectively, and 2 rainbow trout (SE not estimated because all fish were caught on Pass 1) $150 \mathrm{~mm}$ and longer (Table 1 and Appendix B). Another $100 \mathrm{~m}$ sample section located at river $\mathrm{km} 22.5$ supported an estimated 4 (SE not calculated due to all fish captured on Pass 1), 39 (SE: 1.8), and 27 (SE: 0.2); while an upper $105 \mathrm{~m}$ long section supported an estimated 1 (SE not calculated due to all fish captured on Pass 1), 25 (SE: 0.7), and 29 (SE: 0.2) WCT $<75 \mathrm{~mm}, 75-149 \mathrm{~mm}$, and $150 \mathrm{~mm}$ and longer, respectively (Table 2 and Appendix B). Average lengths of WCT ranged from 124 to $165 \mathrm{~mm}$ in the nine South Fork sample sections, while the average length of the two rainbow trout captured in the lowermost section was $230 \mathrm{~mm}$ (Table 2). WCT ranged in length from 50 to $292 \mathrm{~mm}$ (Table 2 and Figure 20).

Habitat surveys were conducted at $\mathrm{km} \mathrm{19.3,22.5} \mathrm{and} \mathrm{25.7.} \mathrm{At} \mathrm{km} 19.3$ the streambed was comprised primarily of sand with sand and silt making up about $55 \%$ of the streambed's surface, while boulder and cobble made up about $35 \%$ (Table 3). At $\mathrm{km} 22.5$ large gravel and cobble dominated the substrate with sand and silt making up 25\% and at $\mathrm{km} 25.7$ sand again dominated with sand and silt making up 50\% and large gravel $25 \%$ of the streambed's surface. Small and large woody debris was relatively scarce in these sample sections with none of the small and from 12 to $75 \%$ of the large debris crossing the wetted channel (Table 3). Much of the large debris that crossed the channel existed in debris aggregates that created excellent pool habitats. Spawning habitat appeared to be moderately abundant at all three sample sections (Table 3). Pool habitats made up $25 \%$ by number and about $20 \%$ by length of all habitat types at $\mathrm{km} 19.3$; 


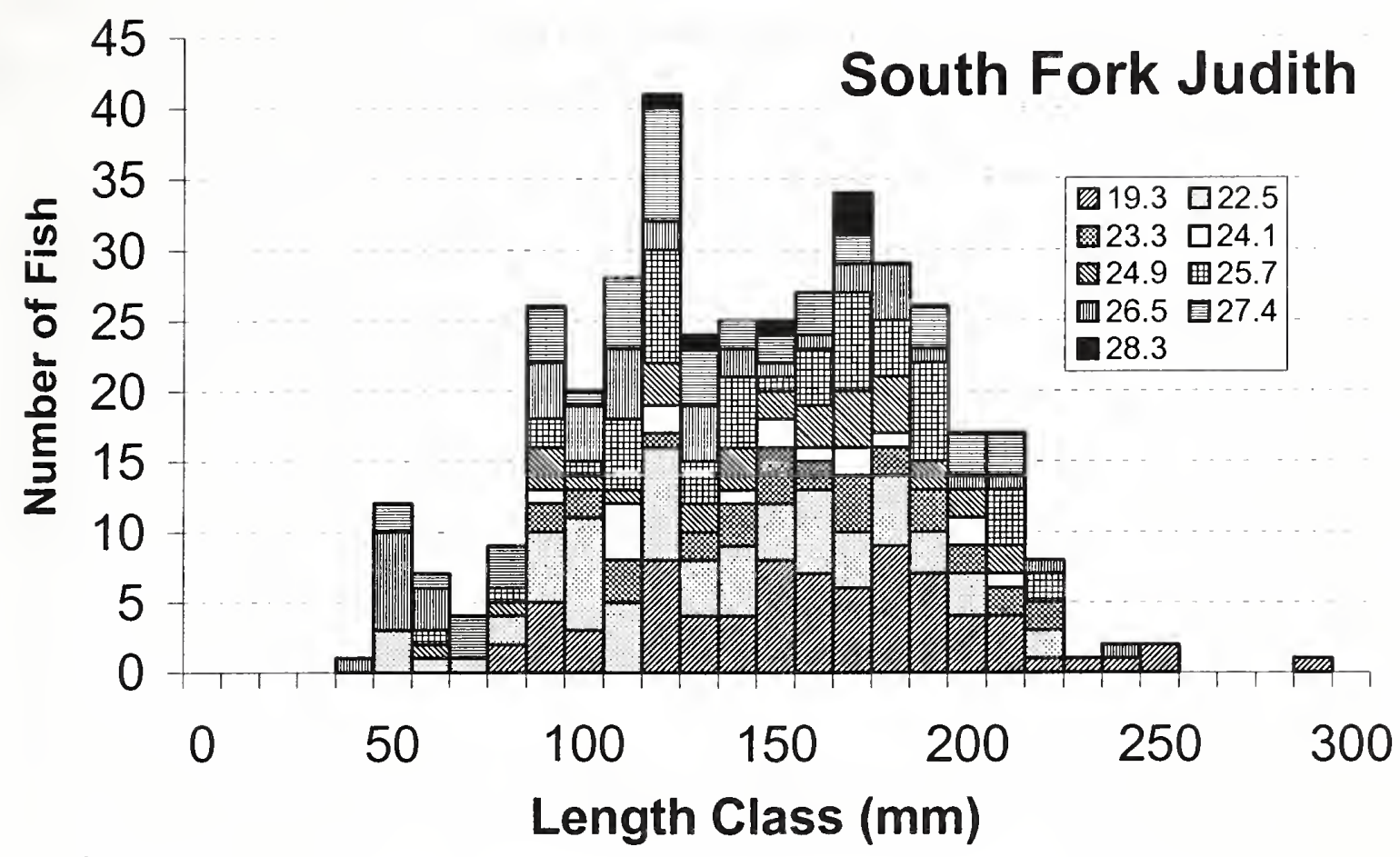

Figure 20. Length frequencies for westslope cut throat trout in nine sections of the South Fork Judith River (by stream kilometer) during late June 2000.

$25 \%$ by number and $18 \%$ by length at $\mathrm{km} 22.5$; and $38 \%$ by number and $31 \%$ by length at $\mathrm{km}$ 25.7 (Table 4). Wetted width averaged 5.6, 4.0, $2.6 \mathrm{~m}$ and depths averaged 35, 26, and $24 \mathrm{~cm}$ in

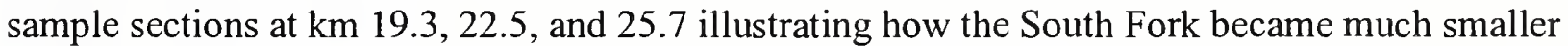
near its headwaters (Table 4). Pools were relatively large, with averaged volumes of from 6.9 to $22.7 \mathrm{~m}^{3}$, in a downstream direction. Residual pool volumes at $\mathrm{km} 22.5$ and 25.7 were 5.9 and $2.5 \mathrm{~m}^{3}$, respectively. Average depths of pools ranged from 33 to $44 \mathrm{~cm}$. Ranks were not assigned to bank cover, bank stability, pool quality, or riparian use at km 19.3. Instream cover was ranked as moderately low at $\mathrm{km} \mathrm{19.3,} \mathrm{but} \mathrm{was} \mathrm{moderately} \mathrm{high} \mathrm{at} \mathrm{both} \mathrm{km} 22.5$ and 25.7 (Table 5). Bank cover and stability were high at $\mathrm{km} 22.5$ and 25.7. Pool quality was moderate and riparian use low at both $\mathrm{km} 22.5$ and 25.7.

\section{Smith Creek}

Smith Creek is about $4.7 \mathrm{~km}$ long and enters the South Fork from the north near river km 18.8 (Figures 1 and 2). A large unnamed tributary enters Smith Creek from the west at about km 1.5 or $0.7 \mathrm{~km}$ below the South Fork Road. Two old beaver ponds just above the South Fork Road impound the creek. Above these ponds the channel gradient is moderate and the valley is comprised primarily of a lodgepole pine forest and is relatively unconfined. The channel above the ponds was believed too small to support fish and improper livestock grazing had led to poor channel conditions. From these ponds downstream the channel gradient increases and the valley 
becomes much more confined. The streambed contained a high proportion of fine sediments both above and below the South Fork Road. Vegetation along the lower portion of the stream was also dominated by lodgepole pine forest. During the summer of 2000 Smith Creek's channel was nearly dry from the beaver ponds down to about stream kilometer 0.5 . Riffle habitats were dry, but some water remained in pool habitats. Fish were observed at high densities in those pools that had water. The field crew believed that some pools that retained water might retain enough water through the summer to provide a refuge and allow some of these fish to survive. Below stream kilometer 0.5 Smith Creek progressively regained surface water flow and aquatic habitat condition improved. A thermograph placed near the mouth of Smith Creek recorded relatively cool water temperatures throughout the summer of 2000 with average daily temperatures seldom exceeding $14 \mathrm{C}$ and maximum temperatures seldom exceeding $17 \mathrm{C}$ (Figure 21).

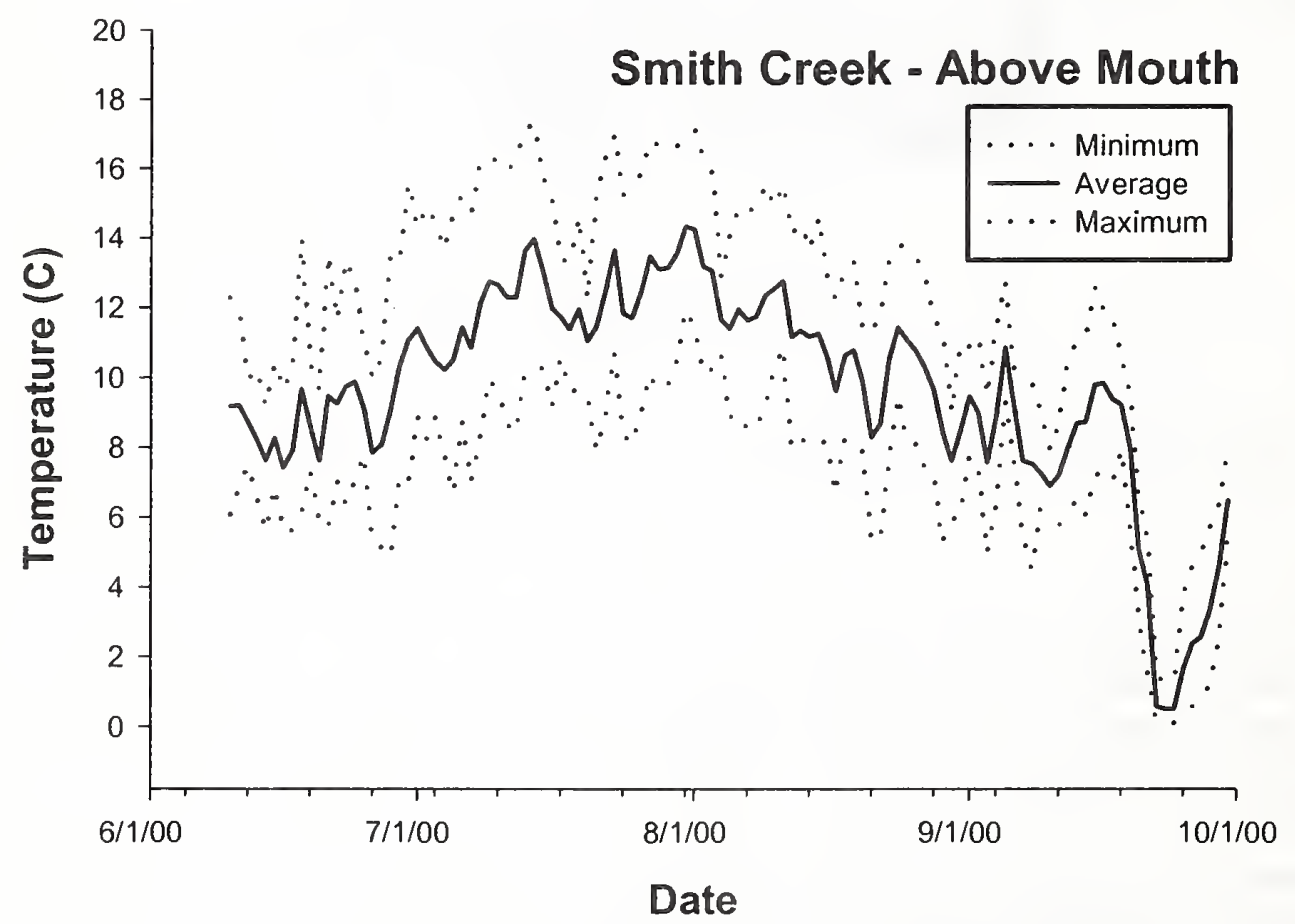

Figure 21. Average, minimum, and maximum water temperatures in Smith Creek just above its mouth during the summer of 2000 .

Smith Creek supports WCT and fish that appeared to be hybrids. A total of 13 WCT and 4 fish identified as hybrids were captured in a single $50 \mathrm{~m}$ section located at $\mathrm{km} 0.8$. As mentioned above, this section was within the portion of stream that was nearly dry. Consequently, all fish were captured in the few pool habitats that had retained water within the section. Captured WCT averaged $132 \mathrm{~mm}$ while hybrids averaged $153 \mathrm{~mm}$ (Table 2). A cursory sample of the stream in 1999 near the South Fork Road found a few fish in this location that also appeared to be hybrids (personal communication, Anne Tews, Montana, Fish, Wildlife and Parks, Lewistown, Montana). 


\section{Discussion}

\section{Fish Distribution and Abundance}

WCT were widely distributed in the upper South Fork Judith River basin and they were generally abundant where they occurred. Relative abundance for WCT generally ranged from 20 to 50 fish $75 \mathrm{~mm}$ and longer per $100 \mathrm{~m}$ of stream length and was as high as nearly $70 \mathrm{WCT}$ per $100 \mathrm{~m}$ of stream. Median relative abundance for WCT $75 \mathrm{~mm}$ and longer was 16.9 per $100 \mathrm{~m}$ in 489 sample sections in the upper Missouri River basin where WCT existed in allopatry and where at least $50 \mathrm{~m}$ of stream length was sampled (Figure 22). WCT in the South Fork Judith basin were generally distributed as far up into headwaters as stream size would support fish. Nonnative rainbow trout occupied the lower portions of Bluff Mountain and Cabin creeks, most of Smith Creek, and were also found in the South Fork Judith as far upstream as river km 19.3, just above Smith Creek. I am unsure how numerous rainbow trout are in the South Fork or how long they have been present in the upper portions of the basin, but their presence so far up the basin is a major concern for WCT conservation and restoration. There was no obvious reason for the presence of brook trout in Bluff Mountain Creek and their absence elsewhere in the upper drainage.

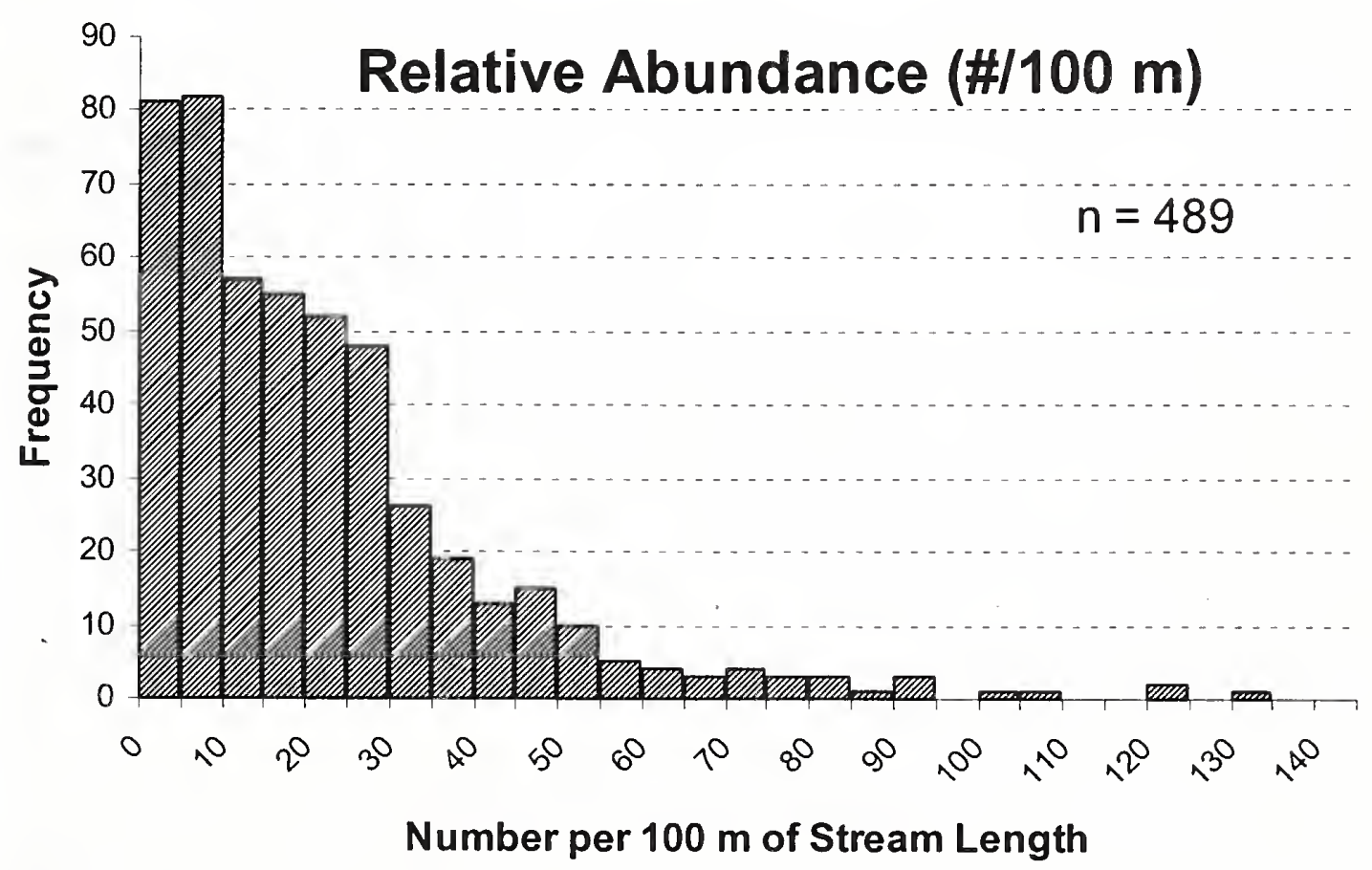

Figure 22. Frequencies of relative abundance (number of fish $75 \mathrm{~mm}$ and longer per $100 \mathrm{~m}$ of stream length) for westslope cutthroat trout in allopatry in 489 sample sections that were a minimum of $50 \mathrm{~m}$ long within the streams of the upper Missouri River basin and a few streams in the upper Clark Fork basin of Montana. 


\section{Recommendations for Conservation of Westslope Cutthroat Trout}

The upper South Fork Judith River basin above Bluff Mountain Creek represents a good opportunity to restore a moderately large connected stream and river system for WCT. The upper South Fork basin from Bluff Mountain Creek upstream contains approximately $60 \mathrm{~km}$ of stream with approximately $40 \mathrm{~km}$ now inhabited by fish. Any attempt to conserve WCT in the upper South Fork will require construction of a fish barrier to eliminate the continuing invasion of brook and rainbow trout into the upper basin. Unfortunately, rainbow trout have already made their way up into the basin at least as far as Smith Creek. It also appears that WCT populations in several tributaries, including Russian and Cabin creeks, have already been slightly hybridized with either rainbow trout or Yellowstone cutthroat trout based on earlier genetic sampling. Fin clips taken for PINE genetic analyses during this study will provide more conclusive evidence for the genetic status of all WCT populations. Lastly, fish identified as either rainbow or hybrids between rainbow and WCT were captured in Bluff Mountain, Cabin, and Smith creeks, as well as the South Fork Judith. Thus, several decisions will have to be made regarding barrier location(s) and either management of existing slightly hybridized populations as conservation populations that could not be used as donors for other waters, or removal of existing hybridized populations and replacing these existing hybrid populations using genetically pure sources from other streams in the basin, either by natural dispersal or by active human intervention.

\section{Acknowledgements}

Matt Sloat and David Barnes collected most of the field data and entered that data into computer data files. Mike Enk of the Lewis and Clark National Forest and Anne Tews of Montana Fish, Wildlife and Parks and their field crews assisted with data collection, logistics, background information, and valuable suggestions. Dr. Robert White and Dee Topp of the Montana Cooperative Fishery Research Unit at Montana State University provided administrative and logistical support.

\section{References}

Environmental Systems Research Institute, Inc. 1999. Arcview GIS program, Version 3.2.

Lisle, T.E. 1987. Using residual depths to monitor pool depths independently of discharge. Research Note PSW-394, USDA Forest Service, Pacific Southwest Forest and Range Experiment Station, Berkley, California.

Lyons, J. 1992. The length of stream to sample with a towed electrofishing unit when fish species richness is estimated. North American Journal of Fisheries Management 12:198203.

Riley, S. C. and K. D. Fausch. 1992. Underestimation of trout population size by maximumlikelihood removal estimates in small streams. North American Journal of Fisheries Management 12:768-776. 
Tews, A., M. Enk, S. Leathe, W. Hill, S.Dalbey, and G. Liknes. 2000. Westslope cutthroat trout (Oncorhynchus clarki lewisi) in northcentral Montana: status and restoration strategies. Montana, Fish, Wildlife and Parks, Great Falls, Montana.

White, G. C., D. R. Anderson, K. P. Burnham, and D. L. Otis. 1982. Capture-Recapture and Removal Methods for Sampling Closed Populations. LA-8787-NERP. UC-11. U.S. Department of Energy, Los Alamos National Laboratory, Los Alamos, New Mexico.

Van Deventer, J. S. and W. S. Platts. 1985. A Computer Software System for Entering, Managing, and Analyzing Fish Capture Data From Streams. Research Note INT-352. U.S. Department of Agriculture, Forest Service, Intermountain Research Station, Ogden, Utah. 
Appendix A1. Number of fish from which fin clips taken for PINE genetic analyses from streams in the upper South Fork Judith River basin, by stream mile and date, during the summer of 2000.

\begin{tabular}{|c|c|c|c|c|}
\hline Stream & KM & Date & $\mathrm{RBxCT}$ & WCT \\
\hline BIG HILL CR & 0.80 & $06 / 12 / 00$ & & 25 \\
\hline BLUFF MOUNTAIN CR & 0.80 & $07 / 24 / 00$ & & 5 \\
\hline BLUFF MOUNTAIN CR & 1.61 & $07 / 21 / 00$ & & 5 \\
\hline BLUFF MOUNTAIN CR & 4.02 & $07 / 26 / 00$ & & 5 \\
\hline BLUFF MOUNTAIN CR TRIB & 0.80 & $07 / 24 / 00$ & & 5 \\
\hline BLUFF MOUNTAIN CR TRIB & 1.61 & $07 / 24 / 00$ & & 3 \\
\hline CABIN CR & 0.80 & $07 / 26 / 00$ & & 5 \\
\hline CABIN CR & 1.61 & $06 / 25 / 00$ & & 5 \\
\hline CABIN CR & 2.41 & $07 / 25 / 00$ & & 5 \\
\hline CABIN CR & 4.83 & $07 / 26 / 00$ & & 5 \\
\hline CABIN CR & 5.63 & $07 / 26 / 00$ & & 5 \\
\hline CROSS CR & 0.80 & $07 / 25 / 00$ & & 24 \\
\hline DEADHORSE CR & 0.80 & $07 / 25 / 00$ & & 5 \\
\hline DEADHORSE CR & 2.41 & $07 / 25 / 00$ & & 5 \\
\hline DEADHORSE CR & 3.22 & $07 / 25 / 00$ & & 5 \\
\hline DEADHORSE CR & 4.02 & $07 / 25 / 00$ & & 5 \\
\hline DEADHORSE CR & 4.83 & $07 / 25 / 00$ & & 5 \\
\hline RUSSIAN CR & 0.80 & $06 / 14 / 00$ & & 5 \\
\hline RUSSIAN CR & 2.41 & $06 / 14 / 00$ & & 5 \\
\hline RUSSIAN CR-W FK TRIB & 0.40 & $06 / 14 / 00$ & & 10 \\
\hline S FK JUDITH R & 22.53 & $06 / 15 / 00$ & & 6 \\
\hline S FK JUDITH R & 26.55 & $06 / 13 / 00$ & & 10 \\
\hline S FK JUDITH R & 27.35 & $06 / 13 / 00$ & & 5 \\
\hline S FK JUDITH R & 28.32 & $06 / 12 / 00$ & & 1 \\
\hline SMITH CR & 0.80 & $07 / 25 / 00$ & 3 & 13 \\
\hline
\end{tabular}




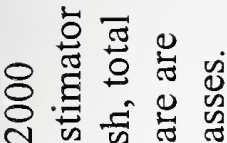
on \& 도웡 于

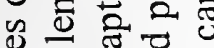

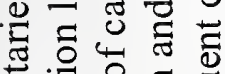
㣢熍 的践 을 현

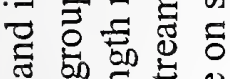

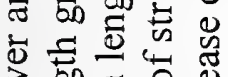
政 을

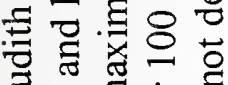
客

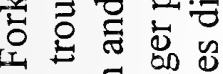

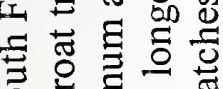

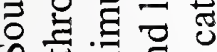

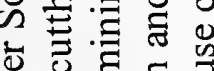
嗐 을 a응 0 o 过它递 ㄱII

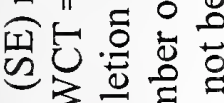
o 응 政 녕 㢦 क

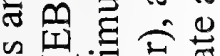
过 응 o 年 응

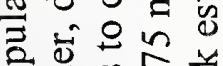
ㅎㅇㅇ 2. $\therefore$ 의

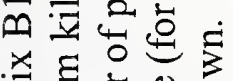

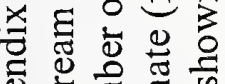

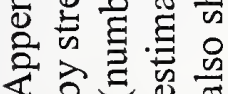

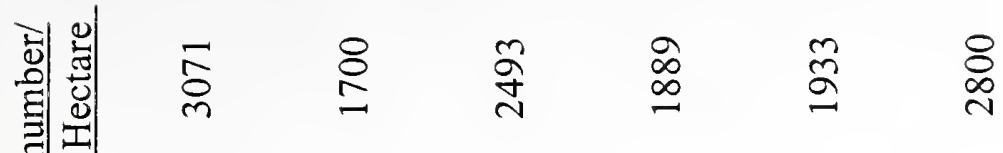

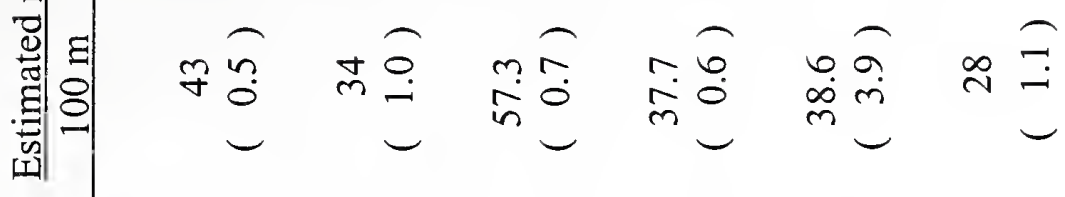

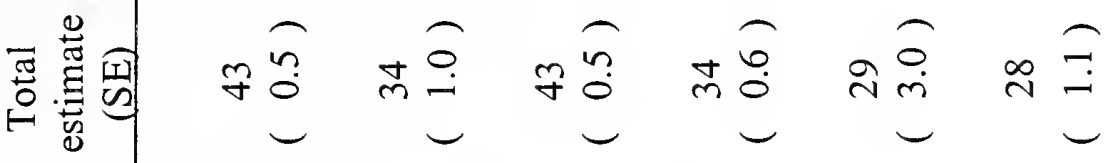

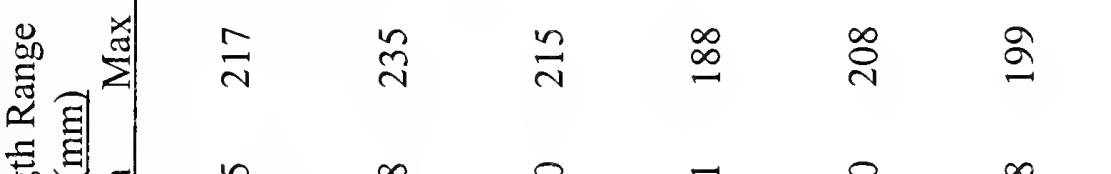

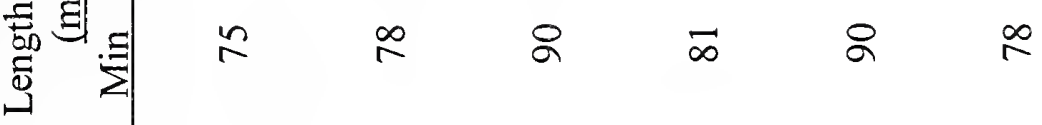

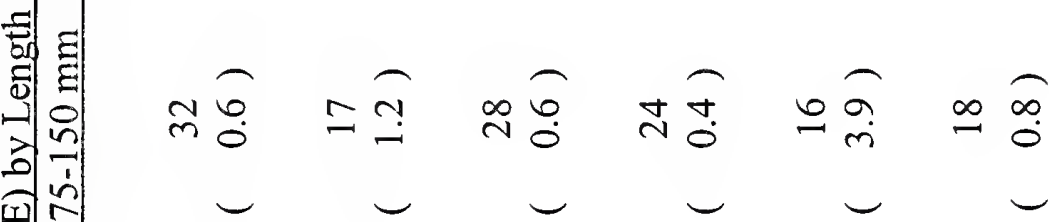

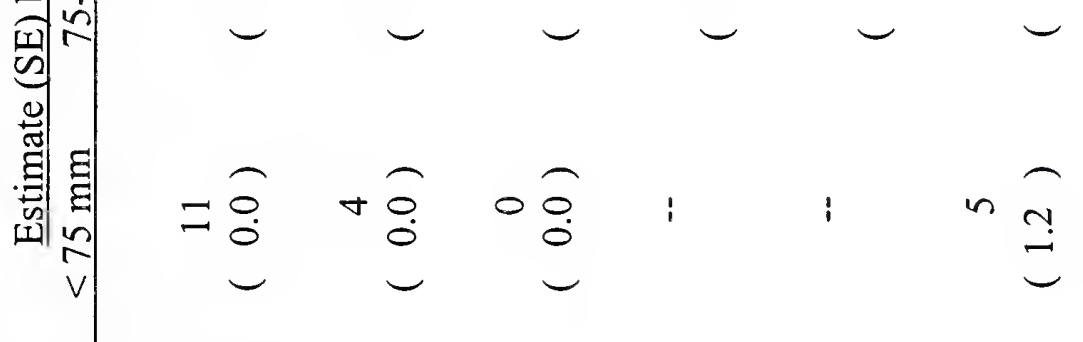

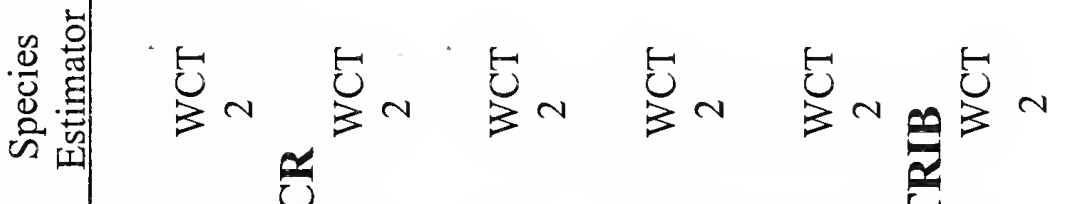

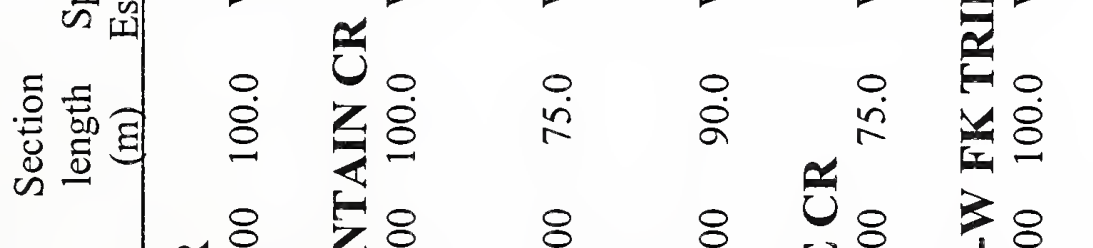

ร

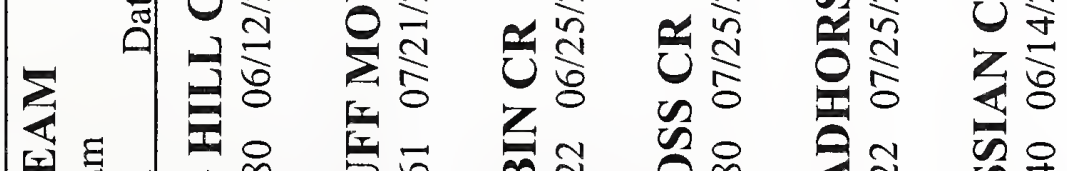

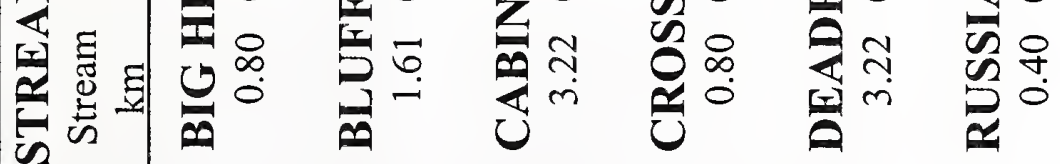




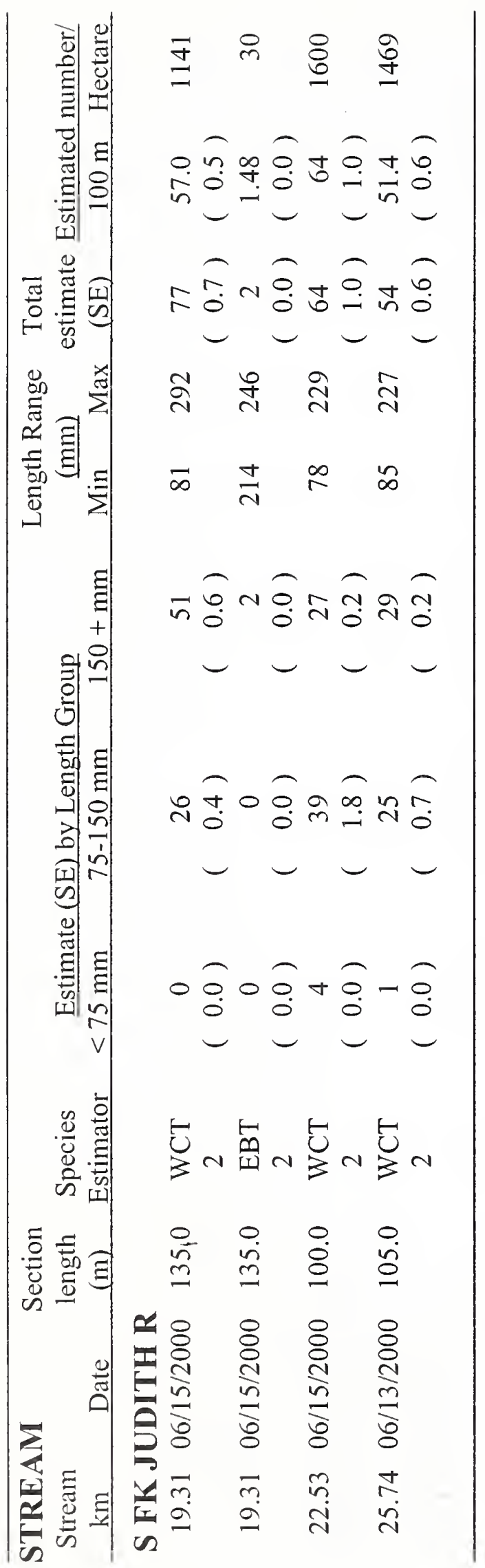




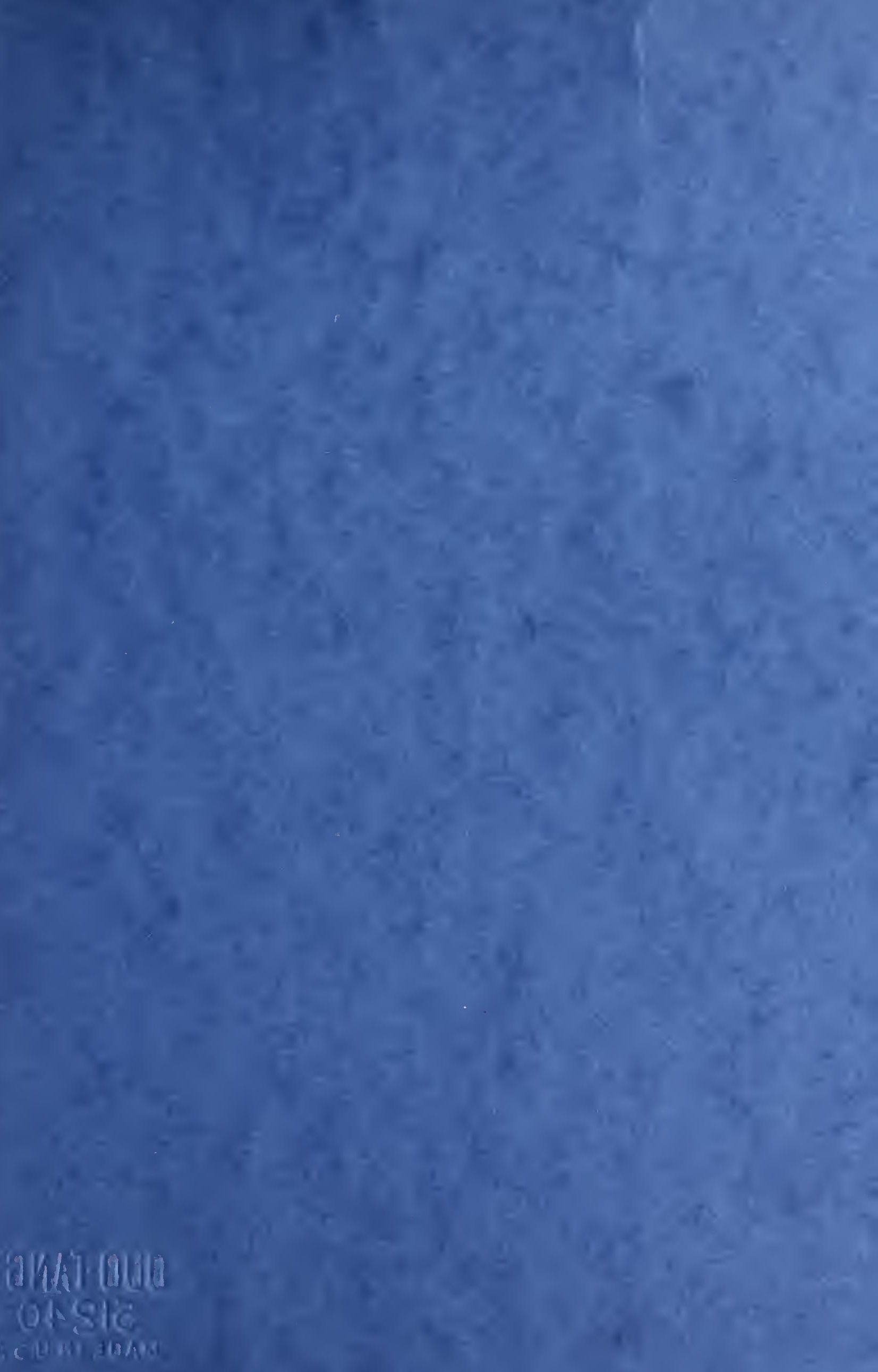


